\title{
Apparent and effective mechanical properties of linear matrix-inclusion random composites: Improved bounds for the effective behavior
}

\author{
Moncef Salmi ${ }^{\mathrm{a}}$, François Auslender ${ }^{\mathrm{a}, *}$, Michel Bornert $^{\mathrm{b}}$, Michel Fogli ${ }^{\mathrm{a}}$ \\ a Mechanical Engineering Research Group, Blaise Pascal University/IFMA, BP 265, 63175 Aubière, France \\ ${ }^{\mathrm{b}}$ Laboratoire Navier, Université Paris-Est, Ecole des Ponts ParisTech, Champs-sur-Marne, 77455 Marne-la-Vallée Cedex, France
}

\section{A R T I C L E I N F O}

\section{Article history:}

Received 25 July 2011

Received in revised form 30 December 2011

Available online 9 February 2012

\section{Keywords:}

Apparent behavior

Effective behavior

Bounds

Linear elasticity

Matrix-inclusion composites

Random microstructure

\begin{abstract}
A B S T R A C T
This paper is devoted to the derivation of improved bounds for the effective behavior of linear elastic matrix-inclusion composites based on a strategy which is inspired by both the works of Huet (1990) and Danielsson et al. (2007). As shown by the former author, the effective properties of random linear composites can be bounded by ensemble averages of their apparent elastic moduli defined on square (or cubic) volume elements (VEs) and computed with either affine displacement Boundary Conditions (BC) or uniform traction BC. However, in the case of a large contrast of the constituents, the discrepancy between the upper and lower bounds remains significant, even for large values of the VE size. This occurs because the contribution to the total potential (or complementary) energy of the particles (or pores) which intersect the edges of the VE becomes unphysically very large when uniform $\mathrm{BC}$ are directly applied to the particles. To avoid such limitations, we considerer non-square (or non-cubic) VEs consisting in simply connex assemblages of cells, each cell being composed of an inclusion surrounded by the matrix, thus forbidding any direct application of BC to the particles. Such VEs are generated by extending the scheme proposed by Danielsson et al. (2007) in the context of periodic random microstructures to fully random microstructures. By applying the classical energy bounding theorems to the non-square VEs, new bounds for the effective behavior are derived. Their application to a two-phase composite composed of an isotropic matrix and aligned identical fibers randomly distributed in the transverse plane leads to sharper bounds which converge quickly with the VE size, even for infinite contrasts.
\end{abstract}

(c) 2012 Elsevier Ltd. All rights reserved.

\section{Introduction}

Most materials, either natural (minerals, biological tissues, etc.) or designed by man (composites, steels, concrete, etc.) are heterogeneous. Efficient modeling approaches are required to determine in a deductive way the global behavior of such a material, to be used for structural calculations at a larger scale, from the local behavior of its various constitutive phases and their spatial distribution. For that, one implicitly or explicitly assumes the following separation of length scales:

$d \ll \ell \ll L_{\text {struct }}$

where the microscale $d$ is the typical size of the heterogeneities of the constituents, $L_{\text {struct }}$ is the typical length of the fluctuations of the mechanical fields generated in the macroscopic structure under consideration if it would be made of a homogeneous material, and the mesoscale $\ell$ is some intermediate length, over which these mac-

\footnotetext{
* Corresponding author. Tel.: +33 473288124; fax: +33 473288027.

E-mail addresses: moncef.salmi@ifma.fr (M. Salmi), francois.auslender@univbpclermont.fr (F. Auslender), michel.bornert@enpc.fr (M. Bornert), michel. fogli@ univ-bpclermont.fr (M. Fogli).
}

roscopic mechanical fields can be considered as uniform, and at the same time, such that volume elements (VE) of size $\ell$ are representative of all microstructural features of the material. This assumption allows to define an effective mechanical behavior such that structural calculations, performed on a fictitious structure with same macroscopic geometry and boundary conditions but made of a homogeneous material exhibiting this behavior, provide, at a much lower numerical cost, macroscopic strain and stress fields which coincide, at least to some accuracy, with the local mechanical fields developed inside the actual heterogeneous structure averaged over volume elements of size $\ell$. The smallest volume element ensuring such a property is called the Representative Volume Element (RVE). Many methods to determine the effective behavior from the properties of the constitutive phases and the microstructure within a RVE have been proposed and can be split into two families.

The first one groups so-called homogenization methods or mean field theories. They are described in many reference extended papers or textbooks among which Willis (1981), Sanchez-Palencia and Zaoui (1987), Nemat-Nasser and Hori (1993), Ponte Castañeda and Suquet (1997) and Bornert (2001). Such methods have now reached a high level of efficiency, especially for linear materials (e.g. Beran, 1968; Willis, 1977; Christensen and Lo, 1979; Ponte Cas- 
tañeda and Willis, 1995; Bornert et al., 1996) but also for nonlinear elastic or viscoplastic composites (e.g. Ponte Castañeda and Suquet, 1997; Ponte Castañeda, 2002; Lahellec and Suquet, 2004) as well as for composites with nonlinear 'hereditary' behaviors, which exhibit a dependence of their current response on the loading path (e.g. Masson and Zaoui, 1999; Lahellec and Suquet, 2007; Doghri et al., 2010). Mean field methods provide either rigourous bounds or estimates of the effective behavior. Usually, bounds can be derived when statistical informations about the phase distribution are available such as some of the $n$th order moments of the characteristic functions $\chi^{r}$ - defined in Section 2.1 - which serve to describe the microstructure. For linear elastic random heterogeneous materials which are focused on in this paper, such informations allow to derive the well-known Voigt (1889) and Reuss (1929) first-order bounds, the Hashin and Shtrikman (1963) second-order bounds and the Beran (1968) higher-order bounds. If the informations about the microstructure are rather of 'morphological' type - e.g. when one phase plays a particular role as it is the case for matrixinclusion composites or when all the phases have the same influence as for granular composites - direct estimates of the effective properties can be proposed such as the well-known Mori and Tanaka (1973) or self-consistent (Kroner, 1958) estimates. When the available informations pertain to both the statistics of the phase distribution and the morphology of the composite, very efficient estimates or bounds for the effective behavior such as the Ponte Castañeda and Willis (1995) estimate or the Morphologically Representative Pattern (MRP) bounds and self-consistent estimates (Stolz and Zaoui, 1991; Bornert et al., 1996) are available.

However, all mean field theories are based on random composites with an infinite extension, for which the condition (1) is exactly satisfied. Mean field estimates or bounds are therefore asymptotic estimates or bounds. Furthermore, they are well suited to predict the behavior of composites with simple ideal model microstructures. For heterogeneous materials with more complex microstructures, when detailed microstructural informations are available in the form of appropriate morphological models or representative images of the microstructure, full-field simulations provide an alternative and nowadays efficient way to evaluate more accurately the effective behavior. This approach consists in computing numerically the apparent behavior of the composite on different VEs of same finite size and approximating the effective behavior as the ensemble average of the apparent behavior. Such an estimate of the effective behavior crucially depends on the size of the VEs, the number of realisations used to evaluate ensemble averages and the prescribed boundary conditions, in addition to the properties of the constitutive phases and the microstructure. Its convergence towards the effective behavior is obtained, at some accuracy, when the size of the samples reaches the size of the RVE, this latter depending on the investigated effective property, the material characteristics such as the contrast between the phases, the volume fractions, the spatial distribution of the phases, etc. and on the expected accuracy. Accordingly, in order to obtain a precise estimate of the effective behavior it becomes essential to evaluate the size of the RVE associated with a particular mechanical or physical property for a given material. However, this size can be very large for many materials, thus leading to excessive computational expense to evaluate the effective behavior from samples of that size. For these materials, we need rigorous criteria able to evaluate how far ensemble averages of the apparent behavior are from the actual effective behavior of the random media when the numerical simulations are performed on samples the size of which is smaller than the RVE size. This problem has been first tackled by Huet (1990) and later by Sab (1992). By making use of different arguments, both these authors independently showed that the arithmetic ensemble average of the apparent stiffness associated with Affine Displacement Boundary Conditions (ADBC) and the harmonic ensemble average of the apparent stiffness associated with Uniform Traction Boundary Conditions (UTBC), both applied to square or cubic shaped samples of size $L$, are upper and lower bounds of the effective behavior, respectively (see Eq. (14)). Further, they proved that the upper bounds (resp. the lower bounds) are decreasing (resp. increasing) functions of the nondimensional size $\delta=L / d$ of the VE. The gap between the upper and lower bounds allows to evaluate how far these bounds are from the actual effective behavior. It has been checked on different applications, investigated for instance by Ostoja-Starzewski (1998) and Ostoja-Starzewski (2006), that the bounding property (14) is able to provide good estimates of the effective behavior for relatively low values of $\delta$ but only for weak to medium contrast between the constituents of the composite. For composites with large contrast, e.g. rigid particles or pores, simulations performed on planar linear elastic disk-matrix composites have revealed that the discrepancy between the upper and lower bounds remains significant even for large values of $\delta$. Later on, numerical simulations carried out by Jiang et al. (2001) again on planar linear elastic disk matrix composites showed that the ensemble average of the apparent behavior associated with periodic BC converges much faster towards the effective behavior than with affine displacement or uniform traction BC. This property has been used by Kanit et al. (2003) who developed an efficient method to evaluate the effective properties based on the notion of integral range and confidence interval. This method allows to predict the number of samples of size $\delta$ necessary to reach a given level of accuracy for the wanted effective property and conversely to predict the relative accuracy for a given number of simulations. However, as pointed out by these authors, the method is efficient only if the size of the samples is larger than the RVE size associated with periodic BC, this latter being defined as the size for which the mean value of the apparent properties of a given material for periodic BC does not evolve any more with the size $\delta$ of the samples. For smaller sizes, the ensemble averages of the apparent properties are potentially biased estimates of the effective properties. As noticed by the authors, this bias is limited by the difference between Huet's bounds, which might however not be accurate for composites with high contrasts, as mentioned above.

The main objective of this work is to provide new sharper bounds of the effective behavior derived from the apparent behaviors as initiated by Huet (1990) and Sab (1992) but by removing the aforementioned limitations observed for uniform strain or stress BC, that is the blow up of the upper bound for rigidly reinforced composites and the vanishing of the lower bound for porous materials. The key point is to notice that this blow up (resp. vanishing) occurs because the part of the strain energy (resp. complementary energy) due to the rigid particles (resp. pores) which intersect the edges of the cell becomes very large when ADBC (resp. UTBC) are directly applied to these rigid particles (resp. pores). To avoid such limitations, we consider non-square (or non-cubic for 3D microstructures) VEs made of assemblages of cells composed of an inclusion located strictly inside the cell and surrounded by the matrix (e.g. Voronoï cells), thus forbidding any direct application of boundary conditions to particles. Such VEs can be generated by extending the scheme proposed by Danielsson et al. (2007) in the context of periodic random microstructures to fully random microstructures. By making use of the classical bounding theorems for linear elasticity and appropriate averaging procedures, sharper bounds of the effective behavior are derived from ensemble averages of the apparent behaviors associated with non-square (or non-cubic) VEs.

The structure of this paper is as follows. The approach initiated by Huet to derive upper and lower bounds for the effective properties from ensemble averages of the apparent properties is briefly recalled in Section 2. The new procedure allowing to derive 
improved bounds for the effective properties by extending Huet's approach to non-square (or non-cubic) VEs for linear matrix-inclusion random composites is presented in Section 3. Special attention is given to the fact that the considered random VEs have a non constant volume. Its applications to a two-phase composite composed of a matrix and aligned identical cylindrical fibers randomly distributed in the transverse plane is depicted in Section 4. The results are presented in Section 4 and compared to the classical Huet's bounds for several contrasts ranging from rigid particles to porous ones and for different inclusion volume fractions. Conclusions are summarized in Section 6.

The tensor notation used herein is a fairly standard one. The orders of the tensors are clear when taken in context. Products containing dots denote summation over repeated latin indices. For example, $L: \varepsilon=L_{i j k l} \varepsilon_{k l} e_{i} \otimes e_{j}$ and $E:: F=E_{i j k l} F_{k l i j}$ where $\left(e_{i}, i=1,3\right)$ is a orthonormal cartesian basis and $\otimes$ denotes the tensor product. On probabilistic aspects, all the random quantities (variables, fields) considered in this work are assumed to be defined on the same probability space $\{\Theta, F, P\}$, where $\Theta$ is a sample space, $F$ is a $\sigma$-algebra of subsets of $\Theta$ and $P$ is a probability on $F$. Moreover, the following notations will be used: if $\xi=(\xi(\omega), \omega \in \Theta)$ is a random variable defined on $\{\Theta, F, P\}$, for any fixed $\omega \in \Theta, \xi(\omega)$ is a $\omega$-realization of $\xi$ (i.e. the realization of $\xi$ associated with the particular event $\omega$ ). For the sake of simplicity, and if no mistake in interpretation is to be feared, the notation $\xi(\omega)$ will also be used to denote such a random variable. In the same way, if $\psi=(\psi(x, \omega), x \in \Delta, \omega \in \Theta)$ is a random field defined on $\{\Theta, F, P\}$ and indexed on $\Delta \subseteq \mathbb{R}^{\mathrm{d}}\left(\mathrm{d} \in \mathbb{N}^{*}\right)$, for any fixed $\omega \in \Theta$, the function $x \rightarrow \psi(x, \omega)$ is a $\omega$-realization of $\psi$ and, to simplify the notations, $\psi(x, \omega)$ will be used to denote just as well this realization as the random field itself. Finally, the statistical expectation and standard deviation of any random variable $\xi$ will be denoted $E(\xi)$ and $\sigma(\xi)$, respectively.

\section{Classical bounds for the apparent and effective behaviors}

In this section we briefly recall some important and classical results on the apparent and effective properties of random linear elastic composites.

\subsection{Definition of the apparent behavior}

Let $A$ be a microstructure characterized by a set of stochastic fields $B \in\left\{\chi^{r} \in\{0,1\}^{\mathbb{R}^{\mathrm{d}} \otimes \Theta}, 1 \leqslant r \leqslant K: \Sigma_{r=1}^{K} \chi^{r}=1\right\}$ where $K$ is the number of phases, $\mathrm{d}=2$ or 3 is the space dimension and $\chi^{r}$ the random characteristic functions, being 1 if the position vector $x$ is in phase $r$, and 0 otherwise. In what follows, for the sake of simplicity, every microstructure (e.g. A) will be identified with the set of functions which characterizes it (i.e. $B$ ). Furthermore, only stationary and ergodic microstructures will be considered. From the microstructure $B$ we obtain for each $\omega \in \Theta$ a realization $B(\omega)$ of the composite. It is worth noting that for two-phase composites only one characteristic function, e.g. the matrix one's $\chi^{M}$, is enough to characterize the microstructure. In order to define square VEs as considered in Huet's approach, let us introduce, in 2D-space, square domains $\Omega_{\delta, X}^{s}$ of centers $X=\left(x_{1}, x_{2}\right)$ and side length $L=\delta d$ given by $\Omega_{\delta, X}^{s}=\left[x_{1}-L / 2, x_{1}+L / 2\right] \times\left[x_{2}-L / 2, x_{2}+L / 2\right]$. A square VE $B_{\delta, X}^{s}(\omega)$ of the composite centered on $X$ and of size $\delta$ is therefore characterized by the restriction of the realization of the composite $B(\omega)$ to the square domain $\Omega_{\delta, X}^{s}$, i.e. $B_{\delta, X}^{s}(\omega) \in\left\{\chi^{r} \in\{0,1\}^{\Omega_{\delta, X}^{s}}, 1 \leqslant r \leqslant K\right.$ : $\left.\sum_{r=1}^{K} \chi^{r}=1\right\}$. The same notation can be used for cubic VEs in 3Dspace, except that $X=\left(x_{1}, x_{2}, x_{3}\right)$ and $\Omega_{\delta X}^{S}=\left[x_{1}-L / 2, x_{1}+L / 2\right] \times$ $\left[x_{2}-L / 2, x_{2}+L / 2\right] \times\left[x_{3}-L / 2, x_{3}+L / 2\right]$. Note that the volume $V_{\delta}=\left|\Omega_{\delta X}^{s}\right|=(d \delta)^{\mathrm{d}}$ of any VE $B_{\delta, X}^{s}(\omega)$ is independent of the realization $\omega$ since the VEs are square or cubic. The spatial average over
$\Omega_{\delta, X}^{s}$ of any random (n-component real-valued) field $\psi(x, \omega)$, e.g. the local strain $\varepsilon(x, \omega)$ and stress $\sigma(x, \omega)$ fields, will be denoted hereafter by

$\langle\psi(\omega)\rangle_{\delta, X}^{s}=\frac{1}{V_{\delta}} \int_{\Omega_{\delta, X}^{s}} \psi(x, \omega) d x$

In what follows, because of the stationarity of the random set $B$, the spatial dependence with respect to the square or cubic window centers $X$ will not be specified unless required. Further, $\partial A$ will denote the outer surface of a set $A$ and $n(x)$ its outward normal at $x \in \partial A$.

When the VE is smaller than the RVE, the behavior of the composite at the mesoscopic scale - namely the behavior of the VE $B_{\delta}^{s}(\omega)$ - called the apparent behavior, depends both on $\omega$ and on the BC prescribed on $\partial \Omega_{\delta}^{s}$. For linear elasticity in small strain framework, the apparent behaviors associated with ADBC $u(x, \omega)=\bar{\varepsilon} . x, \forall x \in \partial \Omega_{\delta}^{s}-$ or to UTBC - $\sigma(x, \omega) \cdot n(x)=\bar{\sigma} \cdot n(x)$, $\forall x \in \partial \Omega_{\delta}^{s}$ - are respectively described by the fourth-order moduli $C_{\delta}^{s, d}(\omega)$ or compliance $S_{\delta}^{s, t}(\omega)$ tensors defined as follows

$\forall \bar{\varepsilon}, \bar{\varepsilon}: C_{\delta}^{s, d}(\omega): \bar{\varepsilon}=\inf _{u \in K_{\delta}^{s}(\bar{\varepsilon})}\langle\varepsilon(u): C(\omega): \varepsilon(u)\rangle_{\delta}^{s}$,

with $K_{\delta}^{s}(\bar{\varepsilon})=\left\{u / u(x)=\bar{\varepsilon} . x \forall x \in \partial \Omega_{\delta}^{s}\right\}$,

$\forall \bar{\sigma}, \bar{\sigma}: S_{\delta}^{s, t}(\omega): \bar{\sigma}=\inf _{\sigma \in S_{\delta}^{s}(\bar{\sigma})}\langle\sigma: S(\omega): \sigma\rangle_{\delta}^{s}$

with $S_{\delta}^{s}(\bar{\sigma})=\left\{\sigma / \operatorname{div}(\sigma(x))=0 \forall x \in \Omega_{\delta}^{s}\right.$;

$\left.\sigma(x) . n(x)=\bar{\sigma} \cdot n(x) \forall x \in \partial \Omega_{\delta}^{s}\right\}$.

In Eqs. (3) and (4) as well as hereafter, the superscripts $d$ and $t$ are associated with ADBC and UTBC, respectively. Further, the superscript $s$ is added to emphasize the fact that the apparent stiffnesses or compliances are defined on square (or cubic) VEs. The tensors $C(x, \omega)$ and $S(x, \omega)=[C(x, \omega)]^{-1}$ are the local stiffness and compliance tensors associated with a $\omega$-realization of the composite, respectively. In what follows, they are assumed to be defined by

$C(x, \omega)=\sum_{r=1}^{K} C^{r} \chi^{r}(\chi, \omega), \quad S(x, \omega)=\sum_{r=1}^{K} S^{r} \chi^{r}(\chi, \omega)$,

where $C^{r}$ (resp. $S^{r}$ ) is the non-random stiffness (resp. compliance) tensor of phase $r$. The displacement, strain and stress fields $u^{d}(x, \omega), \varepsilon^{d}(x, \omega)$ and $\sigma^{d}(x, \omega)$ (resp. $u^{t}(x, \omega), \varepsilon^{t}(x, \omega)$ and $\left.\sigma^{t}(x, \omega)\right)$ solutions of the variational problem (3) (resp. (4)) are also solutions of the following local problem

$$
\left\{\begin{array}{l}
\operatorname{div}(\sigma(x, \omega))=0, \\
\sigma(x, \omega)=C(x, \omega): \varepsilon(x, \omega), \\
\varepsilon(x, \omega))=\frac{1}{2}\left(\nabla u(x, \omega)+{ }^{T} \nabla u(x, \omega)\right)
\end{array}\right\} \quad \forall x \in \Omega_{\delta}^{s}
$$

The apparent moduli $C_{\delta}^{s, d}(\omega)$ and compliance $S_{\delta}^{s, t}(\omega)$ tensors satisfy the relations

$\left\langle\sigma^{d}(\omega)\right\rangle_{\delta}^{s}=C_{\delta}^{s, d}(\omega): \bar{\varepsilon}, \quad\left\langle\varepsilon^{t}(\omega)\right\rangle_{\delta}^{s}=S_{\delta}^{s, t}(\omega): \bar{\sigma}$,

where $\left\langle\sigma^{d}(\omega)\right\rangle_{\delta}^{s}$ and $\left\langle\varepsilon^{t}(\omega)\right\rangle_{\delta}^{s}$ account for the mean values over $V_{\delta}$ of the local fields $\sigma^{d}(x, \omega)$ and $\varepsilon^{t}(x, \omega)$, respectively as defined in Eq. (2). Further, $\left\langle\sigma^{t}(\omega)\right\rangle_{\delta}^{s}=\bar{\sigma}$ and $\left\langle\varepsilon^{d}(\omega)\right\rangle_{\delta}^{s}=\bar{\varepsilon}$.

For ergodic and stationary materials, it has been shown (Papanicolaou and Varadhan, 1978; Sab, 1992) that

$\lim _{\delta \rightarrow \infty} C_{\delta}^{s, d}(\omega)=\lim _{\delta \rightarrow \infty} S_{\delta}^{s, t}(\omega)=C^{\mathrm{eff}}=\left(S^{\mathrm{eff}}\right)^{-1}$,

thus defining the effective behavior $C^{\text {eff }}$ which does not depend on either the BC or the realization $\omega$. In practice, Eq. (8) is satisfied, at a 
specified accuracy, for $\delta$ greater than a critical size $\delta_{R V E}$ which defines the size of the RVE.

\subsection{Order relations for the apparent stiffness and compliance tensors}

Consider, in 2D, a subdivision of $\Omega_{\delta X}^{s}$ into four square-shaped domains $\Omega_{\delta^{\prime}, X^{i}}^{s}$ centered at points $X^{i}=X+( \pm L / 4, \pm L / 4)$ with same size $\delta^{\prime}=\delta / 2$, same volume $V_{\delta^{\prime}, X^{i}}=V_{\delta} / 4$ and such that $\Omega_{\delta, X}^{s}=\bigcup_{i=1}^{4} \Omega_{\delta^{\prime}, X^{i}}^{s}$ In 3D, the subdivision requires eight domains but the proofs are only given in 2D for the sake of simplicity. From the solutions $u_{(i)}^{d}(x, \omega)$ of the local problems (6) defined for arbitrary $\bar{\varepsilon}$ on the subdomains $\Omega_{\delta^{\prime} X^{i}}^{s}$ and associated with ADBC, we define a virtual displacement field $\tilde{u}^{d}(x, \omega)$ on the domain $\Omega_{\delta, X}^{s}$, kinematically admissible with problem (6) associated with ADBC such that its restriction to each subdomain $\Omega_{\delta^{\prime} X^{i}}^{s}$ corresponds to the field $u_{(i)}^{d}(x, \omega)$, i.e. $\tilde{u}^{d}(x, \omega)=u_{(i)}^{d}(x, \omega), \forall x \in \Omega_{\delta^{\prime} X^{i}}^{s}$. The minimal potential energy principle associated with problem (6) with ADBC and applied to $\tilde{u}^{d}(x, \omega)$ can be written

$\left\langle\sigma^{d}(\omega): \epsilon^{d}(\omega)\right\rangle_{\delta, X}^{s} \leqslant\left\langle\tilde{\sigma}^{d}(\omega): \tilde{\epsilon}^{d}(\omega)\right\rangle_{\delta, X}^{s}$

where $\tilde{\epsilon}^{d}(x, \omega)$ and $\tilde{\sigma}^{d}(x, \omega)$ are the kinematically admissible strain and stress fields associated with $\tilde{u}^{d}(x, \omega)$. Applying Hill's lemma to both sides of Eq. (9) and recalling that $V_{\delta^{\prime}, X^{i}}=V_{\delta} / 4$ yields

$\left\langle\sigma^{d}(\omega)\right\rangle_{\delta, X}^{s}: \bar{\epsilon} \leqslant \frac{1}{4} \sum_{i=1}^{4}\left\langle\tilde{\sigma}^{d}(\omega)\right\rangle_{\delta^{\prime}, X^{i}}^{s}: \bar{\epsilon}$.

Definition $(7)_{1}$ of the apparent moduli applied both to VEs $B_{\delta}^{s}(\omega)$ and $B_{\delta, X^{i}}^{s}(\omega)$ leads to

$C_{\delta, X}^{s, d}(\omega) \leqslant \frac{1}{4} \sum_{i=1}^{4} C_{\delta^{\prime}, X^{i}}^{s, d}(\omega)$

where order relations $A \leqslant B$ between two fourth-order tensors $A$ and $B$ should be understood in the sense of quadratic forms, i.e. $t: A: t \leqslant t: B: t$ for any second-order tensor $t$. In view of the assumed stationarity of the material, $E\left(C_{\delta, X}^{s, d}\right)=E\left(C_{\delta}^{s, d}\right)$ and $E\left(C_{\delta^{\prime} X^{i}}^{s, d}\right)=$ $E\left(C_{\delta^{\prime}}^{s, d}\right), \forall i=1, \ldots, 4$; therefore $\forall \delta E\left(C_{\delta}^{s, d}\right) \leqslant E\left(C_{\delta^{\prime}}^{s, d}\right)$ for $\delta^{\prime}=\delta / 2$. Finally, by making use of Eq. (8), we obtain the following order relations and bounds for the effective behavior

$C^{\text {eff }} \leqslant E\left(C_{\delta}^{s, d}\right) \leqslant E\left(C_{\delta^{\prime}}^{s, d}\right) \quad \forall \delta^{\prime}=\delta / 2$.

Similarly, by making use of the complementary energy principle applied to problem (6) (for details see Ostoja-Starzewski (2006)), a hierarchy of upper bounds for the effective compliance is obtained and reads

$S^{\mathrm{eff}} \leqslant E\left(S_{\delta}^{s, t}\right) \leqslant E\left(S_{\delta^{\prime}}^{s, t}\right) \quad \forall \delta^{\prime}=\delta / 2$.

Combining Eq. (12) with (13), it follows

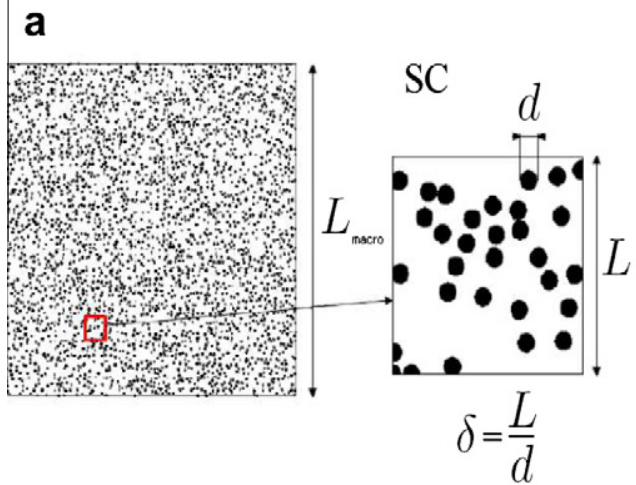

$$
\left(E\left(S_{\delta \prime}^{s, t}\right)\right)^{-1} \leqslant\left(E\left(S_{\delta}^{s, t}\right)\right)^{-1} \leqslant\left(S^{\text {eff }}\right)^{-1}=C^{\text {eff }} \leqslant E\left(C_{\delta}^{s, d}\right) \leqslant E\left(C_{\delta^{\prime}}^{s, d}\right) \forall \delta^{\prime}=\delta / 2 .
$$

The scale-dependant hierarchy of lower and upper bounds for the effective behavior (14) has been first derived by Huet (1990) and later by Sab (1992) but in a different manner. This relation shows that the discrepancy between the upper and lower bounds decreases as $\delta$ increases, until tending towards zero for infinite $\delta$, where the value of the effective behavior is attained. The notations $C_{\delta}^{S C+}=E\left(C_{\delta}^{s, d}\right)$ and $C_{\delta}^{S C-}=\left[E\left(S_{\delta}^{S, t}\right)\right]^{-1}$ will be used to refer to these bounds based on apparent behaviors of 'Square Cells'.

\subsection{Discussion}

As mentioned in Section 1, numerical applications of relation (14) provide good evaluations of the effective properties for situations with small to medium contrast between the phases (Ostoja-Starzewski, 1998). However, for large contrast - e.g., rigid particles or pores - the discrepancy between the upper and lower bounds remains significant even for large value of $\delta$. Illustration of this fact is given in Fig. 2 which reports the evolution of the Huet's bounds of the in-plane effective bulk modulus as functions of $\delta$ for a rigidly-reinforced material and a porous material composed of an isotropic linear elastic matrix and aligned identical cylindrical fibers with diameter $d$ isotropically distributed in the transverse plane. Details on the generation of such microstructures will be given in Section 4.2. Calculations of the apparent behaviors have been performed by means of the Finite Element (FE) software Cast3M (CEA, 2003) coupled with the mesh generator Netgen (Schoberl et al., 2003). For each value $\delta$, the ensemble averages have been evaluated with 2000 different realizations $\omega$ of the microstructure. The average volume fraction of the fibers is $f^{I}=15 \%$ and an isotropic linear elastic behavior has been assigned to the fibers and the matrix, with $k^{I}, \mu^{I}, k^{M}$ and $\mu^{M}$ denoting the bulk and shear moduli of the fibers and the matrix, respectively. The choice $k^{M}=\mu^{M}$ has been made for simplicity. The contrast $c=k^{I} / k^{M}=\mu^{I} / \mu^{M}$ between the phase has been set to $10^{4}$ for the rigidly-reinforced composite and $10^{-4}$ for the porous material. For all numerical applications presented here and later, we have set $\mu^{M}=1$.

The unphysically large (resp. low) values of the upper (resp. lower) bound for the rigidly-reinforced (resp. porous) material occurs because the part of the strain energy due to the particles which intersect the edges of the cell becomes very large when ADBC (resp. UTBC) are directly applied to rigid particles (resp. pores). Thus, by applying unrealistic BC such as a direct enforcement of affine displacement (resp. uniform traction) on the rigid particles (resp. pores), the bounds obtained by Huet (1990) or

Fig. 1. VEs generation from a large microstructure: Square Cell type VE (SC) (a) Voronoï type VE (VOR) (b). 

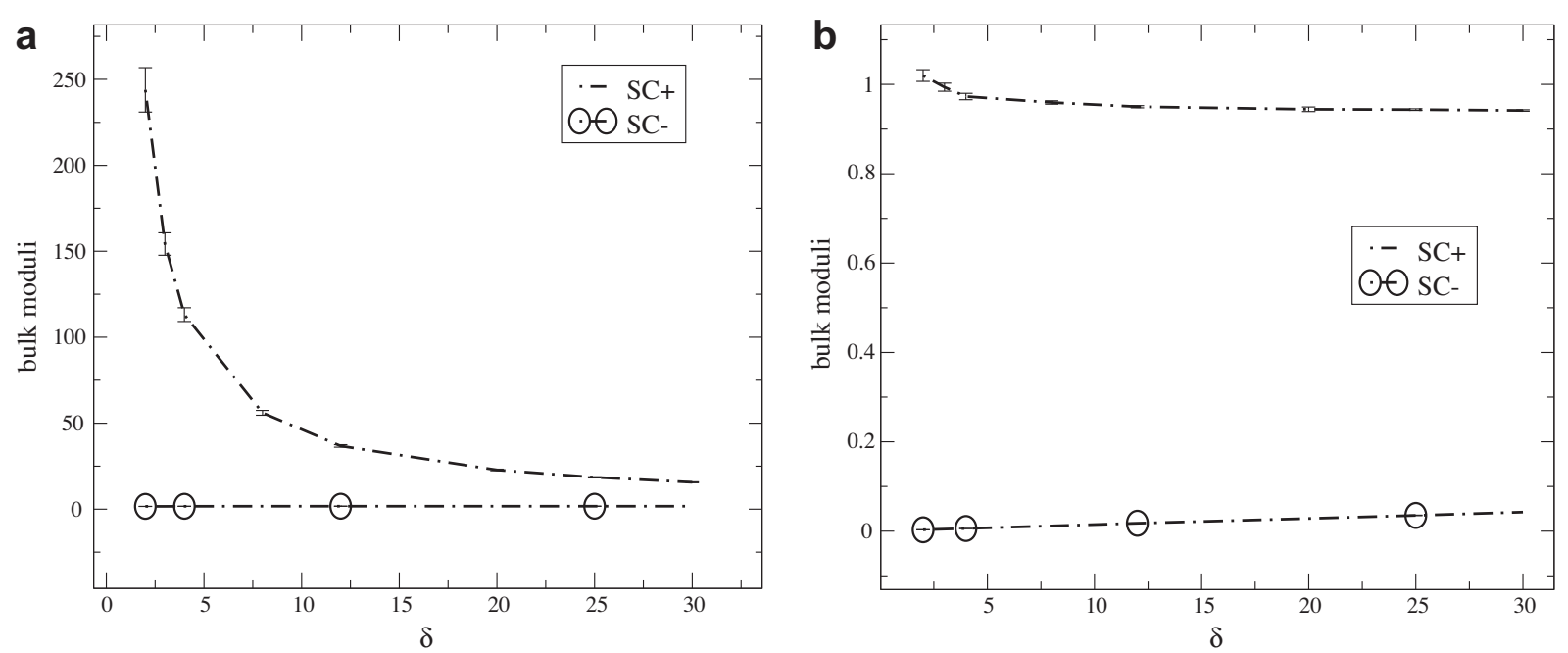

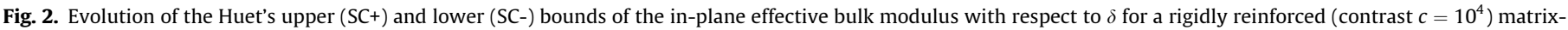
fiber composite (a) and for a porous material $\left(c=10^{-4}\right)(\mathrm{b})$. Fibers volume fraction is $f^{I}=15 \%$.

Sab (1992) strongly overestimate (resp. underestimate) the actual effective properties.

It is worth noting that the evolution of the upper bound as a function of $\delta$ depicted in Fig. 2 can be predicted by a simple model described as follows. In order to account for the part of the strain energy due to the particles which intersect the edges of a square cell of size $L$, the VE $B_{\delta}^{s}(\omega)$ is subdivided into two parts as illustrated in Fig. 3. The first part $B_{\delta}^{(1)}(\omega)$ of domain $\Omega_{\delta}^{(1)}$ and volume $V_{\delta}^{(1)}=4 d(L-d)$ consists of a boundary layer of thickness $d$ such that all the particles which intersect the edges of the VE are included in $B_{\delta}^{(1)}(\omega)$ while the second part $B_{\delta}^{(2)}(\omega)$ of domain $\Omega_{\delta}^{(2)}$ and volume $V_{\delta}^{(2)}=L^{2}-V_{\delta}^{(1)}=(L-2 d)^{2}$ is the core of the VE, namely a square of size $L-2 d$. From this subdivision, we obtain

$$
\begin{aligned}
\bar{\varepsilon} & : C_{\delta}^{s, d}(\omega): \bar{\varepsilon}=\frac{1}{V_{\delta}} \min _{u \in K_{\delta}^{s}(\bar{\varepsilon})} \int_{\Omega_{\delta}^{s}} \varepsilon(u(x)): C(x, \omega): \varepsilon(u(x)) d x \\
& =\frac{1}{V_{\delta}} \min _{u \in K^{(1)}(\bar{\varepsilon})} \int_{\Omega_{\delta}^{(1)}} \varepsilon(u(x)): C(x, \omega): \varepsilon(u(x)) d x \\
& +\frac{1}{V_{\delta}} \min _{u \in K^{(2)}(\bar{\varepsilon})} \int_{\Omega_{\delta}^{(2)}} \varepsilon(u(x)): C(x, \omega): \varepsilon(u(x)) d x \\
& =\bar{\varepsilon}:\left(\frac{V_{\delta}^{(1)}}{V_{\delta}} C_{\delta}^{B L}(\omega)+\frac{V_{\delta}^{(2)}}{V_{\delta}} C_{\delta}^{a p p}(\omega)\right): \bar{\varepsilon}
\end{aligned}
$$

where the sets of kinematically admissible displacement fields $K^{(1)}(\bar{\varepsilon})$ and $K^{(2)}(\bar{\varepsilon})$ are defined by

$$
\begin{aligned}
& K^{(1)}(\bar{\varepsilon})=\left\{u(x) / u(x)=\bar{\varepsilon} . x \forall x \in \partial \Omega_{\delta}^{(1, o u t)} ; u(x)=u^{d}(x, \omega) \forall x \in \partial \Omega_{\delta}^{(1, i n)}\right\}, \\
& K^{(2)}(\bar{\varepsilon})=\left\{u(x) / u(x)=u^{d}(x, \omega) \forall x \in \partial \Omega_{\delta}^{(2)}\right\} .
\end{aligned}
$$

In Eqs. (16), the displacement field $u^{d}(x, \omega)$ corresponds to the solution of the local problem (6) for ADBC. The boundary $\partial \Omega_{\delta}^{(1)}$ is partitioned in two parts $\partial \Omega_{\delta}^{(1)}=\partial \Omega_{\delta}^{(1, \text { out })} \cup \partial \Omega_{\delta}^{(1, \text { in })}(\omega)$ where $\partial \Omega_{\delta}^{(1, \text { in })}=\partial \Omega_{\delta}^{(2)}$ and $\partial \Omega_{\delta}^{(1, \text { out })}=\partial \Omega_{\delta}^{s}$ denote its inner and outer parts, respectively. The tensors $C_{\delta}^{B L}(\omega)$ and $C_{\delta}^{a p p}(\omega)$ defined by Eq. (15) can be interpreted as the apparent stiffness tensors of the boundary layer and the core of the VE, respectively, relative to these specific BC. After taking ensemble averages of this equality and expliciting the volume fractions $\frac{V_{\delta}^{(1)}}{V_{\delta}}$ and $\frac{V_{\delta}^{(2)}}{V_{\delta}}$, one gets

$E\left(C_{\delta}^{s, d}\right)=C_{\delta}^{S C+}=E\left(C_{\delta}^{a p p}\right)+\frac{4}{\delta}\left(1-\frac{1}{\delta}\right)\left(E\left(C_{\delta}^{B L}\right)-E\left(C_{\delta}^{a p p}\right)\right)$.

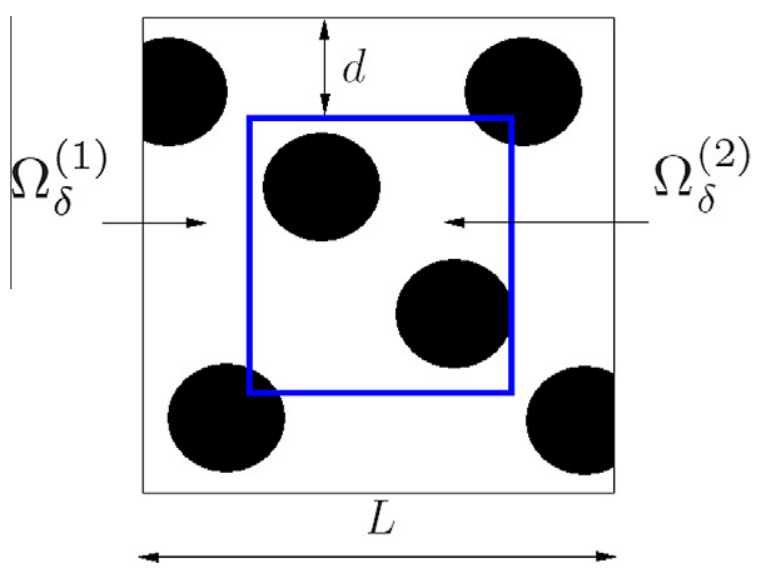

Fig. 3. Subdivision of a square VE associated with the approximate bound $K_{\delta}^{A b}$.

In a first approximation, it can be assumed that the statistical expectation of the apparent behavior of the boundary layer with constant thickness $d$ does only very slightly depend on $\delta$ and that the statistical expectation of the apparent modulus of the core can be evaluated by a modelling approach which does not artificially over-evaluate the effective properties, since it is based on much more realistic boundary conditions. It could for instance be approximated by the bound $E\left(C_{\delta}^{V O R+}\right)$ proposed in Section 3 of this paper. Combining these considerations with (12) and noting in addition that the contribution of the boundary layer is predominant, i.e. $E\left(C^{B L}\right) \gg E\left(C_{\delta}^{a p p}\right)$, one gets:

$C^{\mathrm{eff}} \leqslant C_{\delta}^{S C+} \approx E\left(C_{\delta}^{V O R+}\right)+\frac{4}{\delta}\left(1-\frac{1}{\delta}\right) E\left(C^{B L}\right)$.

This relation can be further simplified by noting that the dependance with $\delta$ of the first term of the second member is likely to be much weaker than that of the second (see Section 3), so that the substitution $E\left(C_{\delta}^{V O R+}\right) \approx E\left(C_{\infty}^{V O R+}\right)=C^{\text {eff }}$ can be made, which is consistent with the limit $E\left(C_{\infty}^{s, d}\right)=C^{\text {eff }}$. The following approximate scaling of $E\left(C_{\delta}^{s, d}\right)$ with $\delta$ is finally obtained:

$$
\begin{aligned}
C^{\mathrm{eff}} & \leqslant C_{\delta}^{S C+} \approx C_{\delta}^{A b}=C^{(1)}+\frac{4}{\delta}\left(1-\frac{1}{\delta}\right) C^{(2)}, \quad \text { with } C^{(1)} \\
& =C^{\mathrm{eff}} \text { and } C^{(2)} \simeq E\left(C^{B L}\right) .
\end{aligned}
$$




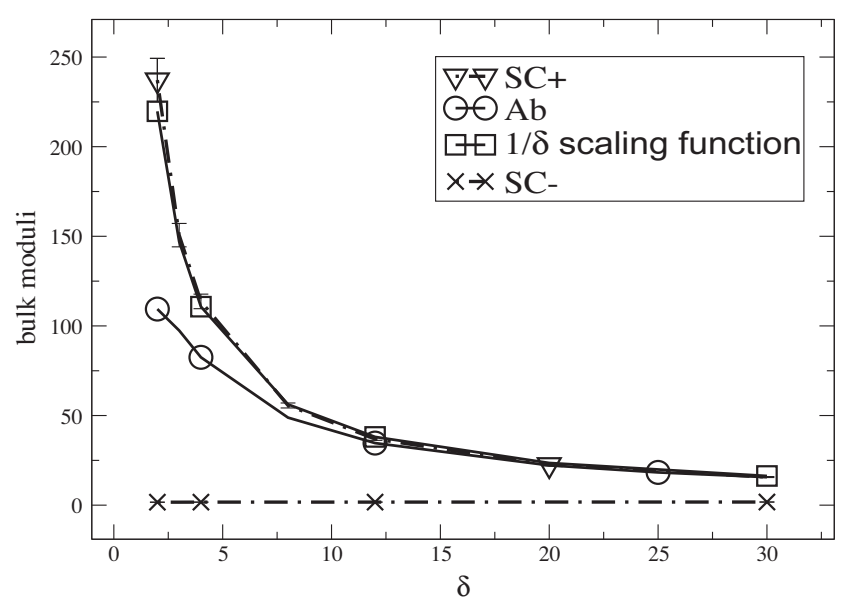

Fig. 4. Rigidly reinforced composite $\left(c=10^{4}, f=15 \%\right)$ : evolution with respect to $\delta$ of the Huet's $(S C+, S C-)$ and approximate $(\mathrm{Ab})$ bounds for the in-plane effective bulk modulus.

A fitting of the in-plane bulk modulus $K_{\delta}^{S C+}$ of the bound $C_{\delta}^{S C+}$ reported in Fig. 2(a) with the approximate bound $(\mathrm{Ab}) K_{\delta}^{A b}=K^{(1)}+\frac{4}{\delta}\left(1-\frac{1}{\delta}\right) K^{(2)}$, where $K^{(1)}$ has been evaluated by $2 K^{(1)}=2 K^{\text {eff }} \approx K_{30}^{S C+}+K_{30}^{S C-}$ allows to determine the constant $K^{(2)} \approx E\left(K^{B L}\right)$ and consequently the whole dependence of $K_{\delta}^{A b}$ on $\delta$. The variations of $K_{\delta}^{A b}$ and $K_{\delta}^{S C+}$ with respect to $\delta$ are reported in Fig. 4. A relatively good agreement between both curves is obtained, thus showing that the evolution of Huet's upper bound $K_{\delta}^{S C+}$ as a function of $\delta$ for rigidly-reinforced materials is essentially due to the predominant contribution of the boundary layer to the apparent behaviors when ADBC are applied. Of course, the approximate bound defined by Eq. (19) should not be understood as a rigorous result but rather as a simplified approach aiming at evaluating in a very simplified way the contribution of the boundary layer which dominates the dependence of the SC + bounds with $\delta$. In this regard, the discrepancy observed for small values of $\delta$ between the $\mathrm{SC}+$ and approximate bounds is probably due to the crude approximation assuming that the apparent behavior of the boundary layer does not depend on $\delta$. To this regard, it is worth noting that the evolution of the upper bound $K_{\delta}^{S C+}$ with respect to $\delta$ can be very well fitted with the simpler scaling law $K^{3} / \delta+K^{\text {eff }}$ as shown in Fig. $4-$ the constant $K^{3}$ being fitted numerically in agreement with $K_{\delta}^{S C+}$ at $\delta=30$.

For the in-plane deviatoric behavior, because of the quadratic anisotropy of Huet's upper bound generated by the square shape of the VEs, the simple approach described by Eq. (19) needs to be slightly modified to fully describe the evolution of $C_{\delta}^{S C+}$. For the sake of clarity, this modified approach will be described in Section 4.5.3 devoted to the specificities induced by square shaped VEs. However, the key point is still that the blow-up of in-plane deviatoric moduli is produced by the particles which intersect the edges of the cells. Note that another closed-form scaling function describing the evolution with respect to $\delta$ of the apparent tensor of moduli namely a nonlinear combination of $K_{\delta}^{S C+1-}$ and $G_{\delta}^{S C+1-}$ - has been obtained for linear elastic random polycrystals by Ranganathan and Ostoja-Starzewski (2008) but does not apply to the present context. Let us finally mention that making use of the classical Voigt upper bound (Voigt, 1889) to estimate the apparent behavior of the boundary layer, i.e. $C^{B L} \approx f^{I} C^{I}+\left(1-f^{I}\right) C^{M}$, results in an approximate upper bound $C_{\delta}^{A b}$ that strongly overestimates Huet's upper bound $C_{\delta}^{S C+}$. Indeed, the assumption made by Voigt's approach which considers that the strain inside the quasi-rigid particles $\left(c=10^{4}\right.$ ) belonging to the boundary layer is uniform and equal to the imposed strain $\bar{\varepsilon}$ is too strong and should be relaxed. Only some components $\varepsilon_{i j}(x)$ of the local strain tensor turn out to be equal to $\bar{\varepsilon}_{i j}$, and that only in a small part of the particles intersecting the edges of the VE.

\section{Improved procedure to bound the effective properties of matrix-inclusion random composites}

To avoid the above-mentioned limitation of Huet's approach namely the blow up or vanishing for high contrast of the bounds of the effective properties generated by a direct application of uniform strain or stress BC on the particles, we describe in this section a new procedure to bound the effective properties of random composites which relies on the construction of non-square (or non-cubic in 3D) VEs whose boundaries do not intersect the particles.

\subsection{Non-square VEs}

In order to design VEs forbidding any direct application of boundary conditions to particles, the random microstructures considered in this paper are of matrix-inclusion type only. It is assumed that no contact occurs between the inclusions which can be of any size and any shape. The new VEs are defined as elementary cell assemblages, each elementary cell being composed of a sole inclusion surrounded by matrix. To generate such VEs, we consider a very large square window of the microstructure $B(\omega)$ - namely a Representative Volume Element (RVE) - and perform a subdivision (e.g. a Voronoï subdivision) of this microstructure into $M$ non-overlapping elementary cells where $M$ is the number of inclusions in this window, such that the union of these cells coincides with the large window. Then, $n$ non-overlapping square windows $\Omega_{\delta, X_{k}}^{s}$ ( $n$ being the number of realizations) of size $\delta$ and centers $X_{k}$ $(k=1 \ldots n)$ are extracted from the RVE. They are used to generate $n$ non-square domains $\Omega_{\delta, X_{k}}(\omega)$ made of the set of elementary cells the centers of which belong to $\Omega_{\delta, X_{k}}^{s}$, the center of each elementary cell being defined as the center of mass of its inclusion. Each VE $B_{\delta, X}(\omega)$ is therefore a restriction of a realization of the composite $B(\omega)$ to its corresponding non-square domain $\Omega_{\delta, X}(\omega)$ derived from the subdivision into elementary cells. Note that the upperscript $s$ introduced in Section 2.1 to denote square VEs is left over for non-square VEs. A classical manner to generate the elementary cell is to subdivise the RVE into $M$ Voronoï cells, each cell being made of a sole inclusion surrounded by matrix (see Fig. 1(b)). Such a way to generate non-square VEs by means of Voronoï cell assemblages can be interpreted as an extension of the scheme proposed by Danielsson et al. (2007) for periodic random microstructures to fully random microstructures. The Voronoï subdivision is well adapted to matrix-inclusion microstructures for which the inclusions are of same shape or size, as it will be the case in the numerical applications presented hereafter. However, for inclusions of different sizes or/and shapes, a more general subdivision should be considered. One instance of subdivision can be derived from the distance function $d\left(x, I_{i}\right)$ between a point $x$ belonging to the RVE and an inclusion $i(i=1, \ldots, M)$ of domain $I_{i}$ defined as $d\left(x, I_{i}\right)=\min _{y \in I_{i}} d(x, y)$ where $d(x, y)$ is the euclidian distance between two points $x, y$. The elementary cell number $i$ is then defined, as by e.g. Bilger et al. (2005), by the set $\Omega_{i}=\left\{x \in R V E, \forall j \neq i, d\left(x, I_{i}\right) \leqslant d\left(x, I_{j}\right)\right\}$, thus characterizing the subdivision thoroughly.

An other example of subdivision suitable for matrix-inclusion composites with particles of different sizes and/or shapes is provided by the Morphologically Representative Pattern -based description of the microstructure developed by Stolz and Zaoui (1991) and Bornert et al. (1996). In this approach, the heterogeneous medium is described as a set of disjoint subdomains that map the whole RVE. It is assumed that these domains may be grouped into families with identical geometries and mechanical properties within the same family. These families are called "Morphologically Representative Patterns" (MRP). The way to define precisely the MRP is reported in Bornert et al. (1996). An example of pattern could be a particle of given size and shape surrounded by a layer of matrix - several possibilities to define its external 
shape are conceivable. The classical Hashin Composite Spheres Assemblage (CSA) (Hashin, 1962) is one example of MRP-based subdivision for a "theoretical" microstructure consisting of spherical inclusions of different sizes embedded in a matrix such that the union of all the patterns, i.e. the composite inclusions, fills the whole RVE. For the CSA, each composite inclusion consists of an inclusion surrounded by a spherical layer of matrix such that the inclusion volume fraction inside the pattern is equal to the inclusion volume fraction of the RVE.

An example of VEs generated by the above-mentioned scheme and issued from a Voronoï subdivision of a matrix-inclusion composite made of randomly distributed fibers of same size and orientation is reported in Fig. 1(b). In 3D-situations, non-cubic VEs can be obtained by the same methodology. In what follows, for the sake of simplicity, only 2D-situations are considered. However, all the obtained results still hold in 3D-situations since they can be enlarged in a straightforward way by making use of non-cubic VEs.

\subsection{New bounds for linear matrix-inclusion random composites}

To derive new bounds for the effective properties, a key point is to notice that the shape and volume of the non-square VEs $B_{\delta}(\omega)$ derived by the methodology described in the previous section depend on the realization $\omega$. Again, because of the stationarity of the random set $B$, the spatial dependence on $X$ is not specified unless required. Accordingly, all the definitions given in Section 2.1 for deterministic domains $\Omega_{\delta}^{s}$ and volumes $V_{\delta}$ associated with square VEs can be enlarged to the non-square domains $\Omega_{\delta}(\omega)$ and their volumes $V_{\delta}(\omega)$ which are now random variables. More precisely, the apparent behaviors associated with ADBC or UTBC are defined by

$\forall \bar{\varepsilon}, V_{\delta}(\omega) \bar{\varepsilon}: C_{\delta}^{d}(\omega): \bar{\varepsilon}=\inf _{u \in K_{\delta}(\bar{\varepsilon}, \omega)} \int_{\Omega_{\delta}(\omega)} \varepsilon(u(x)): C(x, \omega): \varepsilon(u(x)) d x$,

with $K_{\delta}(\bar{\varepsilon}, \omega)=\left\{u(x) / u(x)=\bar{\varepsilon} . x \forall x \in \partial \Omega_{\delta}(\omega)\right\}$,

$\forall \bar{\sigma}, V_{\delta}(\omega) \bar{\sigma}: S_{\delta}^{t}(\omega): \bar{\sigma}=\inf _{\sigma \in S_{\delta}(\bar{\sigma}, \omega)} \int_{\Omega_{\delta}(\omega)} \sigma(x): S(x, \omega): \sigma(x) d x$,

with $S_{\delta}(\bar{\sigma}, \omega)=\left\{\sigma(x) / \operatorname{div}(\sigma(x))=0 \forall x \in \Omega_{\delta}(\omega)\right.$;

$\left.\sigma(x) . n(x, \omega)=\bar{\sigma} \cdot n(x, \omega) \forall x \in \partial \Omega_{\delta}(\omega)\right\}$.

By extending Huet approach to non-square VEs, the following property is obtained:

For non-square VEs such as the ones defined in Section 3.1, $\forall \delta \in \mathbb{R}^{+}$and for $\delta^{\prime}=\delta / 2$, we have

$$
\begin{aligned}
E\left(\frac{V_{\delta^{\prime}}}{E\left(V_{\delta^{\prime}}\right)} S_{\delta^{\prime}}^{t}\right)^{-1} & \leqslant E\left(\frac{V_{\delta}}{E\left(V_{\delta}\right)} S_{\delta}^{t}\right)^{-1} \leqslant\left(S^{\mathrm{eff}}\right)^{-1}=C^{\mathrm{eff}} \leqslant E\left(\frac{V_{\delta}}{E\left(V_{\delta}\right)} C_{\delta}^{d}\right) \\
& \leqslant E\left(\frac{V_{\delta^{\prime}}}{E\left(V_{\delta \prime}\right)} C_{\delta^{\prime}}^{d}\right) .
\end{aligned}
$$

Derivation of (22): For the sake of simplicity, the proof of Eq. (22) is given for a Voronoï subdivision of the microstructure knowing that it still works for more general partitions such as the ones described in the previous section. Similarly to Huet's approach, the VE $B_{\delta, X}(\omega)$ of size $\delta$ and center $\mathrm{X}$ is subdivided into four disjoined sub-VEs $B_{\delta^{\prime} X^{i}}(\omega)$ of size $\delta^{\prime}=\delta / 2$, centers $X^{i}=X+( \pm L / 4, \pm L / 4)$, volume $V_{\delta^{\prime}, X^{i}}(\omega)$, and such that $B_{\delta}(\omega)=\bigcup_{i=1}^{4} B_{\delta^{\prime}, X^{i}}(\omega)$. The VEs $B_{\delta^{\prime}, X^{i}}(\omega)$ are derived in the same way than $B_{\delta, X}(\omega)$, i.e. they are defined as the set of Voronoï cells of the microstructure $B(\omega)$ the centers of which belong to the square domain $\Omega_{\delta^{\prime} X^{i}}^{s}$ of size $\delta^{\prime}$ and centers $X^{i}$. As shown on Fig. $1(\mathrm{~b})$, the VEs $B_{\delta, X}(\omega)$ and $B_{\delta^{\prime} X^{i}}(\omega)$ are non-square and no particle intersects their border. Again, from the solutions $u_{(i)}^{d}(x, \omega)$ of the local problems (6) now defined on the non-square subdomains $\Omega_{\delta^{\prime} X^{i}}(\omega)$ and submitted to ADBC, a virtual displacement field $\tilde{u}^{d}(x, \omega)$ defined on the domain $\Omega_{\delta, X}(\omega)$ and kinematically admissible with problem (6) associated with ADBC is built such that its restriction to each subdomain $\Omega_{\delta^{\prime} X^{i}}(\omega)$ corresponds to the fields $u_{(i)}^{d}(x, \omega)$, i.e. $\tilde{u}^{d}(x, \omega)=u_{(i)}^{d}(x, \omega)$, $\forall x \in \Omega_{\delta^{\prime} X^{i}}(\omega)$. The minimal potential energy principle associated with problem (6) defined on $\Omega_{\delta, X}(\omega)$ for ADBC and applied to $\tilde{u}^{d}(x, \omega)$ leads to Eq. (9) with $\Omega_{\delta, X}^{s}(\omega)$ replaced by $\Omega_{\delta, X}(\omega)$. Applying Hill lemma to both sides of Eq. (9) and making use of the definition (20) of the apparent behavior now applied to both VEs $B_{\delta, X}(\omega)$ and $B_{\delta, X^{i}}(\omega)$ give

$V_{\delta, X}(\omega) C_{\delta}^{d}(\omega) \leqslant \sum_{i=1}^{4} V_{\delta^{\prime}, X^{i}}(\omega) C_{\delta^{\prime}, X^{i}}^{d}(\omega)$.

In view of the assumed stationarity of the material,

$$
\begin{array}{rlrl}
E\left(V_{\delta^{\prime}, X^{i}} C_{\delta^{\prime}, X^{i}}^{d}\right) & =E\left(V_{\delta^{\prime}} C_{\delta^{\prime}}^{d}\right), & & E\left(V_{\delta^{\prime}, X^{i}}\right)=E\left(V_{\delta^{\prime}}\right)=E\left(V_{\delta}\right) / 4 \\
& =E\left(V_{\delta, X}\right) / 4 & \forall i=1, \ldots, 4 .
\end{array}
$$

Averaging inequality (23) and making use of relations (24) yields

$\forall \delta, \quad \frac{E\left(V_{\delta} C_{\delta}^{d}\right)}{E\left(V_{\delta}\right)} \leqslant \frac{E\left(V_{\delta^{\prime}} C_{\delta^{\prime}}^{d}\right)}{E\left(V_{\delta^{\prime}}\right)} \quad$ for $\delta^{\prime}=\delta / 2$

Since Eq. (8) implies

$\lim _{\delta \rightarrow \infty} \frac{E\left(V_{\delta} C_{\delta}^{d}\right)}{E\left(V_{\delta}\right)}=\frac{E\left(V_{\delta \rightarrow \infty} C^{\mathrm{eff}}\right)}{E\left(V_{\delta \rightarrow \infty}\right)}=C^{\mathrm{eff}}$,

we have

$\forall \delta, \quad C^{\mathrm{eff}} \leqslant \frac{E\left(V_{\delta} C_{\delta}^{d}\right)}{E\left(V_{\delta}\right)} \leqslant \frac{E\left(V_{\delta^{\prime}} C_{\delta^{\prime}}^{d}\right)}{E\left(V_{\delta^{\prime}}\right)} \quad$ for $\delta^{\prime}=\delta / 2$.

By the same reasoning but using the complementary energy principle applied to problem (6) now defined on $B_{\delta}(\omega)$ for UTBC, the following new hierarchy of upper bounds for the effective compliance is obtained

$\forall \delta, \quad S^{\mathrm{eff}} \leqslant \frac{E\left(V_{\delta} S_{\delta}^{t}\right)}{E\left(V_{\delta}\right)} \leqslant \frac{E\left(V_{\delta^{\prime}} S_{\delta^{\prime}}^{t}\right)}{E\left(V_{\delta^{\prime}}\right)} \quad$ for $\delta^{\prime}=\delta / 2$.

Combining Eq. (27) with (28) leads to (22), thus ending the proof.

In what follows, for ease of notation, we make use of the definitions

$C_{\delta}^{V O R+}=E\left(\frac{V_{\delta}}{E\left(V_{\delta}\right)} C_{\delta}^{d}\right), \quad C_{\delta}^{V O R-}=E\left(\frac{V_{\delta}}{E\left(V_{\delta}\right)} S_{\delta}^{t}\right)^{-1}$,

for the Voronoï $C_{\delta}^{V O R+/-}$ type upper and lower bounds.

\subsection{Statistical estimation}

The ensemble averages used in the new bounding theorem (22) differ from the ones employed in Huet's bounds (14) since a weight $\frac{V_{\delta}(\omega)}{E\left(V_{s}\right)}$ is now allocated to $C_{\delta}^{d}(\omega)$ (resp. $\left.S_{\delta}^{t}(\omega)\right)$. To estimate these ensemble averages the following methodology is used. As described in Section 3.1, from the large microstructure $B(\omega)$ are extracted $n$ VEs $B_{\delta, X^{i}}(\omega)(i=1 \ldots n)$ each consisting of a Voronoi cells assemblage, the centers of which belong to their corresponding square cell of size $\delta$ and center $X^{i}$. From the ergodic property, each VE $B_{\delta, X^{i}}(\omega)$ can be understood as a $\omega_{i}$-realization $B_{\delta, X}\left(\omega_{i}\right)$ of the microstructure of size $\delta$ and center $X$, defined as the restriction of the $\omega_{i}$-realization $B\left(\omega_{i}\right)$ of the composite to the domain $\Omega_{\delta, X}\left(\omega_{i}\right)$, i.e. $B_{\delta, X}\left(\omega_{i}\right) \equiv B_{\delta, X^{i}}(\omega)$. Again, because of the stationarity property, the dependence on $\mathrm{X}$ is omitted. Considering a $n$-sample $\left(\left(C_{\delta}^{d}\left(\omega_{i}\right), V_{\delta}\left(\omega_{i}\right)\right), \omega_{i} \in \Theta, 1 \leqslant i \leqslant n\right)$ of the couple $\left(C_{\delta}^{d}, V_{\delta}\right)$, the ensemble averages in Eq. (22) can be estimated by 


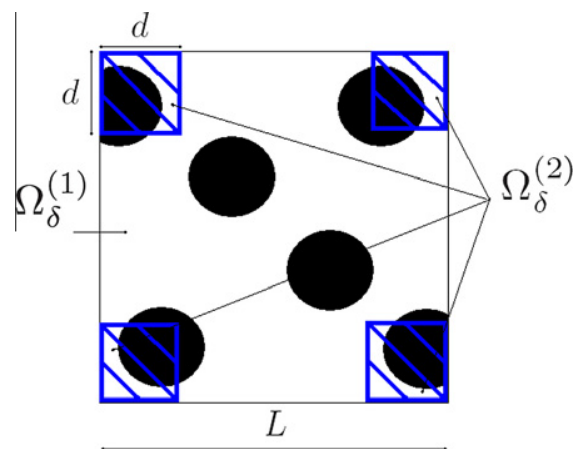

Fig. 5. Subdivision of a square VE associated with the approximate bound $\mu_{\delta}^{A b}$.

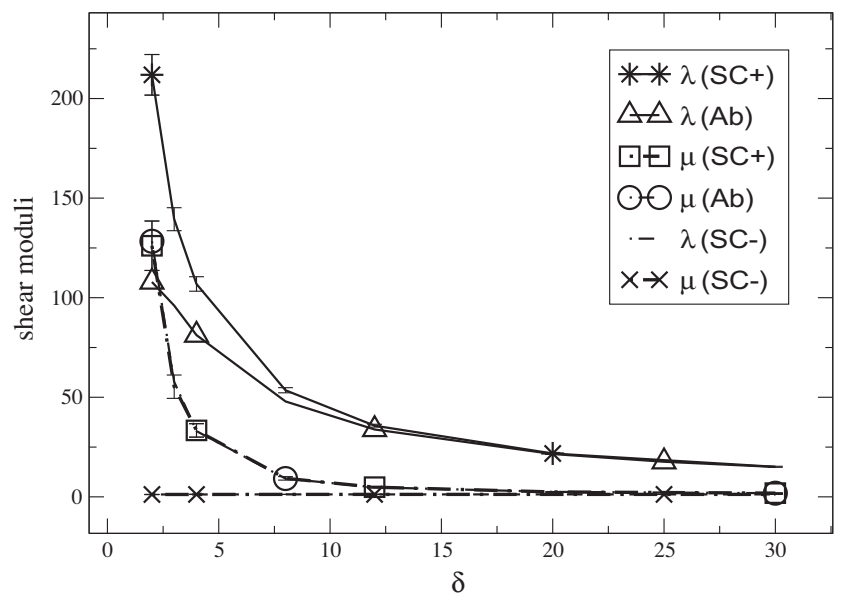

Fig. 6. Rigidly reinforced composite $\left(c=10^{4}, f=15 \%\right)$ : variation of the Huet's $(\lambda(S C+), \mu(S C+))$ and approximate $(\lambda(A b), \mu(A b))$ upper bounds for the in-plane effective shear modulus as functions of $\delta$.

$E_{n}\left(\frac{V_{\delta} C_{\delta}^{d}}{E_{n}\left(V_{\delta}\right)}\right)=\frac{\sum_{i=1}^{n} V_{\delta}\left(\omega_{i}\right) C_{\delta}^{d}\left(\omega_{i}\right)}{\sum_{i=1}^{n} V_{\delta}\left(\omega_{i}\right)}$

where the subscript $n$ means that the expectation is performed by using $n$ realizations of the couple $\left(C_{\delta}^{d}, V_{\delta}\right)$.

A second and perhaps more relevant estimate can be obtained by given a statistical meaning to the weight $\frac{V_{\delta}(\omega)}{E\left(V_{\delta}\right)}$. For that, we still start from the subdivision of the large microstructure $B(\omega)$ into $n$ VEs $B_{\delta}\left(\omega_{i}\right)$ and we consider $N$ points $x_{I}=x\left(\omega_{I}\right), 1 \leqslant I \leqslant N, \omega_{I}$ $\in \Theta, N \gg n$, randomly and uniformly distributed over $B(\omega)$. Then we associate to each point $x_{I}$ the apparent modulus $C_{\delta}^{d}$ (or compli- ance $\left.S_{\delta}^{t}\right)$ of the VE to which the point $x_{I}$ belongs. The mean $E\left(C_{\delta}^{d}\right)$ can then be estimated by

$$
E_{N}\left(C_{\delta}^{d}\right)=\frac{1}{N} \sum_{I=1}^{N} C_{\delta}^{d}\left(\omega_{I}\right)=\frac{\sum_{i=1}^{n} \sum_{j=1}^{n_{\delta}\left(\omega_{i}\right)} C_{\delta}^{d}\left(\omega_{i}\right)}{\sum_{i=1}^{n} \sum_{j=1}^{n_{\delta}\left(\omega_{i}\right)}}=\frac{\sum_{i=1}^{n} n_{\delta}\left(\omega_{i}\right) C_{\delta}^{d}\left(\omega_{i}\right)}{\sum_{i=1}^{n} n_{\delta}\left(\omega_{i}\right)}
$$

where $n_{\delta}\left(\omega_{i}\right)$ is the number of points $x_{I}$ which belong to the VE $B_{\delta}\left(\omega_{i}\right)$. In Eq. (31), we made use of the fact that $C_{\delta}^{d}\left(\omega_{I}\right)=C_{\delta}^{d}\left(\omega_{i}\right)$ for each point $x_{I}$ belonging to $B_{\delta}\left(\omega_{i}\right)$. Since the points $x_{I}$ are uniformly distributed over $B(\omega), n_{\delta}\left(\omega_{i}\right)$ is related to $V_{\delta}\left(\omega_{i}\right)$ by a linear relation $n_{\delta}\left(\omega_{i}\right)=\varrho V_{\delta}\left(\omega_{i}\right)$ where $\varrho$ is the volume density number, i.e. the number of points $x_{I}$ which belong to a unit volume. The scalar $\varrho$ does not depend neither on $\omega_{i}$ nor on $\delta$. Accordingly, Eq. (31) can be rewritten as

$$
E_{N}\left(C_{\delta}^{d}\right)=\frac{1}{N} \sum_{I=1}^{N} C_{\delta}^{d}\left(\omega_{I}\right)=\frac{\sum_{i=1}^{n} V_{\delta}\left(\omega_{i}\right) C_{\delta}^{d}\left(\omega_{i}\right)}{\sum_{i=1}^{n} V_{\delta}\left(\omega_{i}\right)}=E_{n}\left(\frac{V_{\delta} C_{\delta}^{d}}{E_{n}\left(V_{\delta}\right)}\right),
$$

and thus provides another estimate of the ensemble average in Eq. (22). From a theoretical viewpoint, this second approach is more relevant than the first one since we are left with classical expectations where each realization has the same weight. However, from a practical point of view, the expectation, the standard deviation as well as the distribution function of the random variables $C_{\delta}^{d}(\omega), S_{\delta}^{t}(\omega)$ are computed by taking into account the weight $\frac{V_{\delta}\left(\omega_{i}\right)}{E\left(V_{s}\right)}$ associated with each realization $\omega_{i}$. In what follows, for ease of notation, the subscripts $N$ and $n$ used in Eqs. (30)-(32) will be omitted.

\section{Application: transverse response of random linear composites}

The bounding theorem (22) presented in Section 3.2 holds for two or three dimensional matrix-inclusion random composites. However, in order to reduce the numerical cost required to compute the apparent behaviors for each realization, we consider as a first illustration the case of a composite made of a matrix and aligned fibers which can be addressed by means of low cost 2D numerical simulations. For such composites, it becomes possible to compute the ensemble averages of the apparent behaviors by making use of at least 1000 different realizations for each VE size $\delta$ with standard computational power.

\subsection{Material description}

More precisely, the studied material is a two-phase composite consisting of a matrix and aligned identical cylindrical fibers randomly and isotropically distributed in the transverse plane. Each phase is assumed to be isotropic and to follow Hooke's law. The
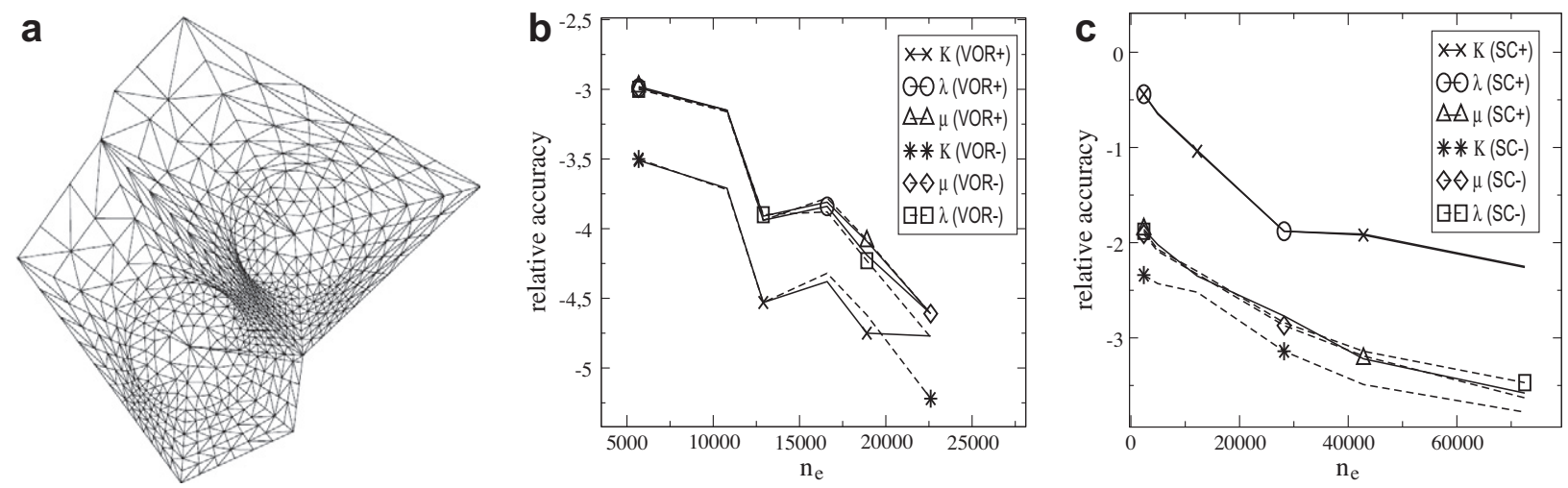

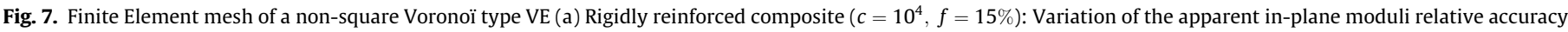
(in decimal $\log$ scale) with respect to the FE number $n_{e}$ for a non-square Voronoï type VE $(\delta=12)$ (b) and for a square VE $(\delta=12)$ (c). 

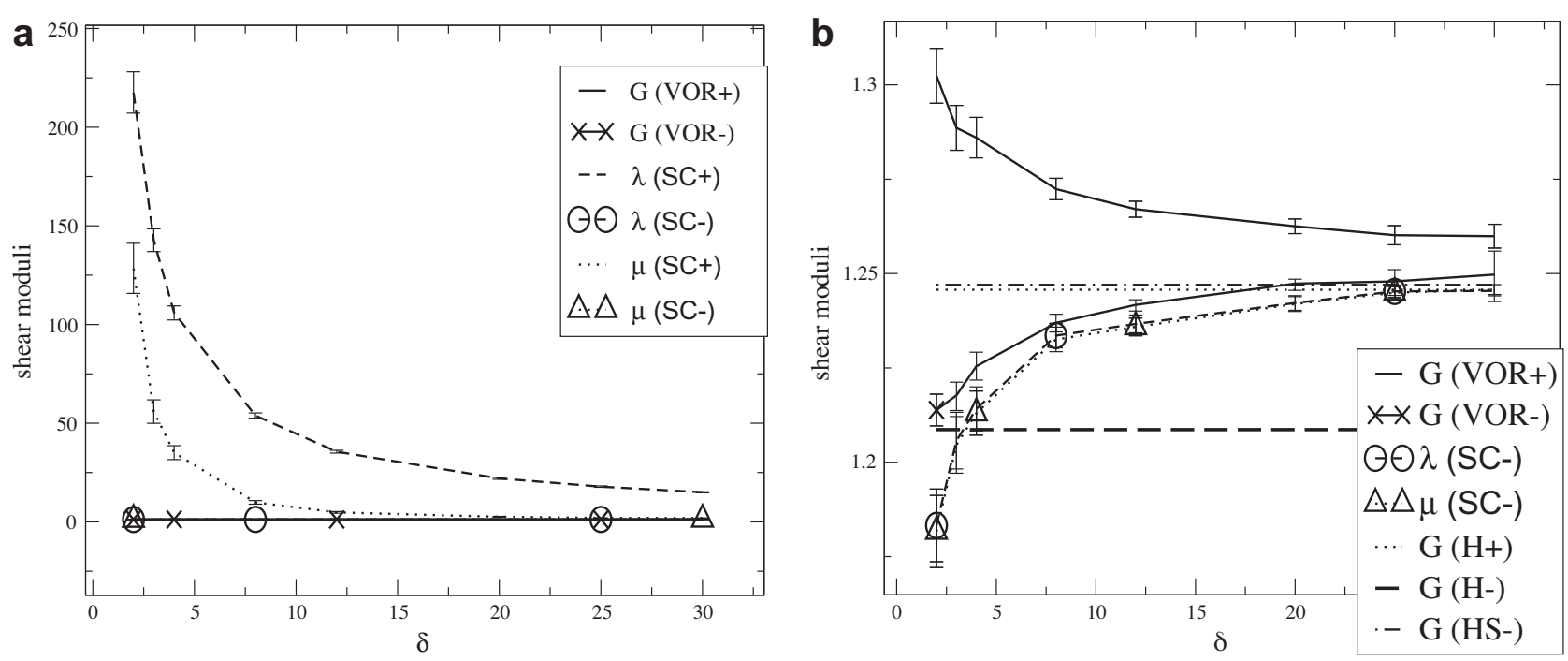

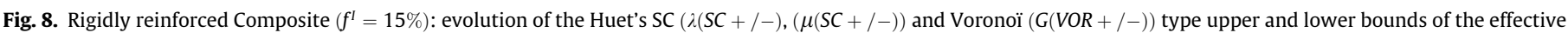
in-plane shear modulus as functions of $\delta$, as well as the Hashin (H) and Hashin and Shtrikman (HS) upper (+) and lower (-) bounds. Full curves (a), enhanced zoom (b).
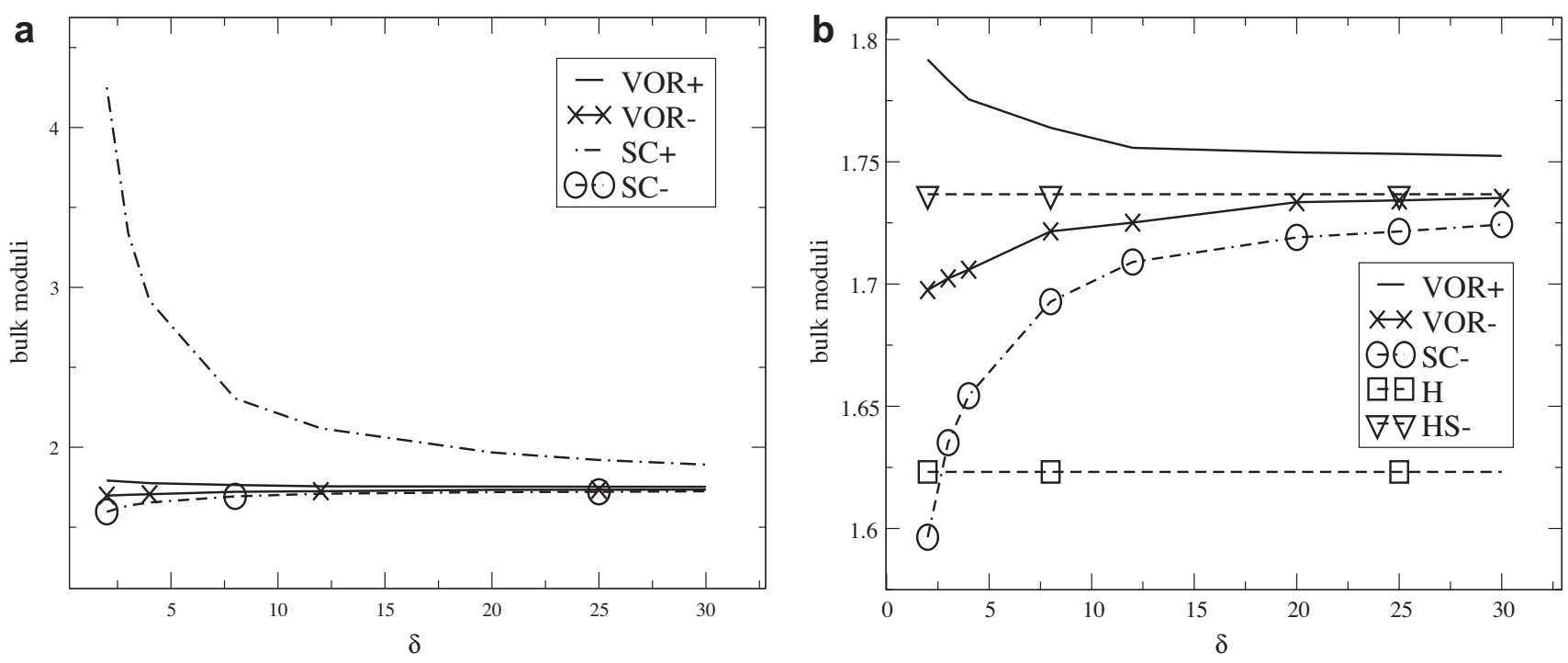

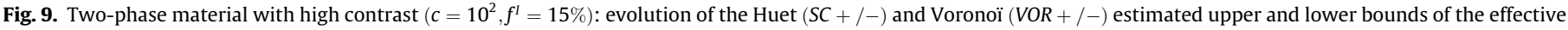
in-plane bulk modulus as functions of $\delta$. Full curves (a), enhanced zoom (b).

bulk and shear moduli of the constituents of the composite are given by $k^{M}=\mu^{M}=1$ for the matrix and $k^{I}=\mu^{I}=c$ for the inclusions. Due to rotational invariance along the fiber axis $e_{3}$ of both the constitutive relations of the phases and the statistics of their spatial distribution, the effective behavior of the linear composite is transversally isotropic. The same property holds true for the upper and lower bounds $C_{\delta}^{V O R_{-}^{+}}$obtained in (22). Indeed, although each realization of the apparent behavior $C_{\delta}^{d}(\omega), S_{\delta}^{t}(\omega)$ is not transversally isotropic since the spatial distribution of the fibers in a finite size VE $B_{\delta}(\omega)$ is not transversally isotropic for $\delta \leqslant \delta_{R V E}$, their ensemble average is transversally isotropic since averaging over the whole set of realizations amounts to average over the whole possible orientations of the microstructure of the VEs, thus leading to an overall transversally isotropic behavior. ${ }^{1}$

\footnotetext{
${ }^{1}$ Rigorously speaking, the VOR bounds still depend on the geometry of the square domains $\Omega_{\delta}^{s}$ by which the Voronoï cells are selected to define the VEs. Therefore, they may keep some kind of quadratic symmetry. But in practice, this anisotropy is so small that it can be neglected.
}

In order to simplify the computations, we only consider the transverse response of the composite. The local problem to be solved can thereby be reduced in plane strain framework to a two-dimensional problem for which the fibers are represented by disks of same diameter $d$ and the VEs are subjected to in-plane loading. Due to the transversally isotropic property of both the effective behavior and its Voronoï type bounds, their in-plane behavior is fully characterized by their in-plane bulk and shear moduli.

\subsection{Microstructure generation}

The microstructure of the material is generated by the following way. The centers of the disks are randomly implemented by a Poisson process in a large window of size $125 d$ containing approximatively 3000 fibers for $f=15 \%$. A constraint on the minimal distance between the disks of same diameter $d$ is imposed to prevent overlapping and contact. In this process, the disk are generated sequentially: if the current disk the position of which is 

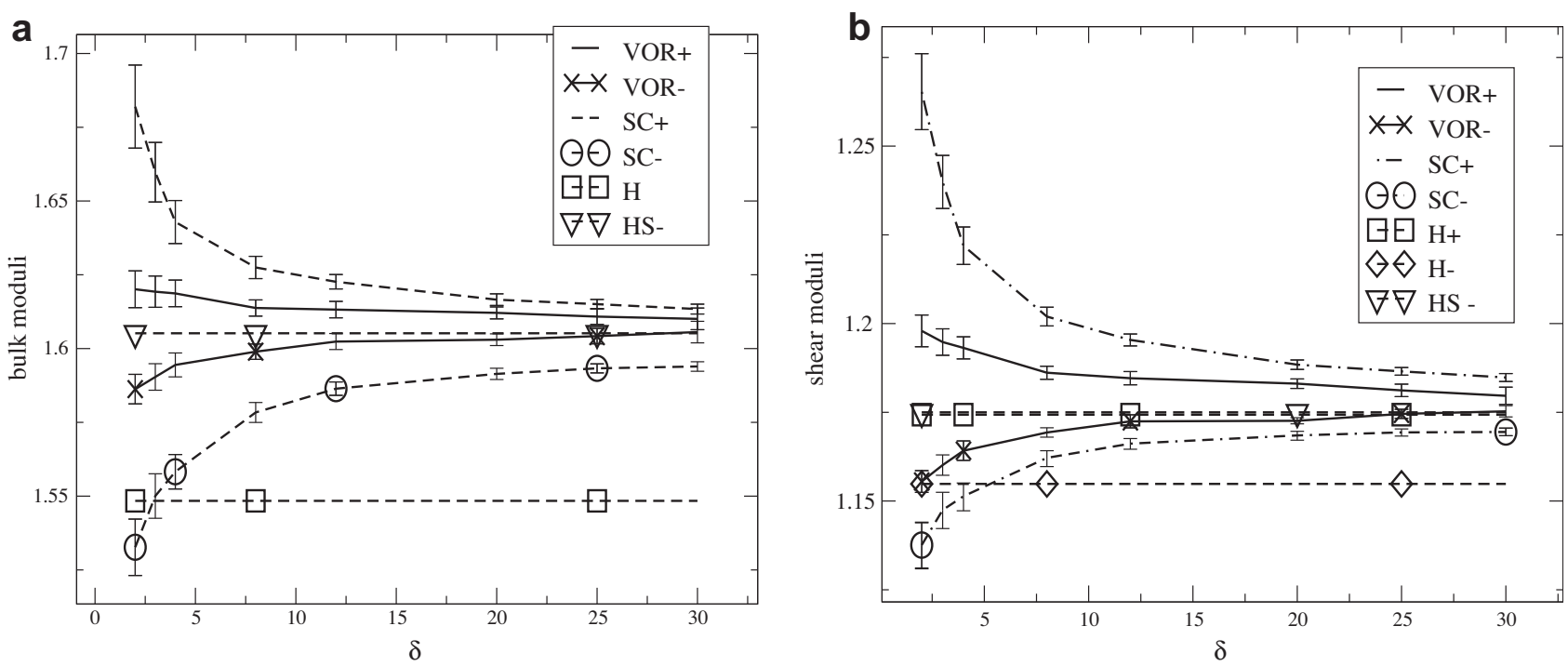

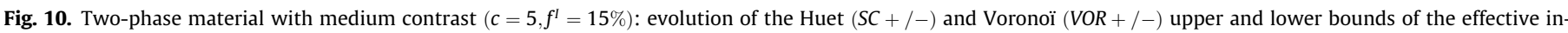
plane bulk modulus (a) and shear modulus (b) as functions of $\delta$.
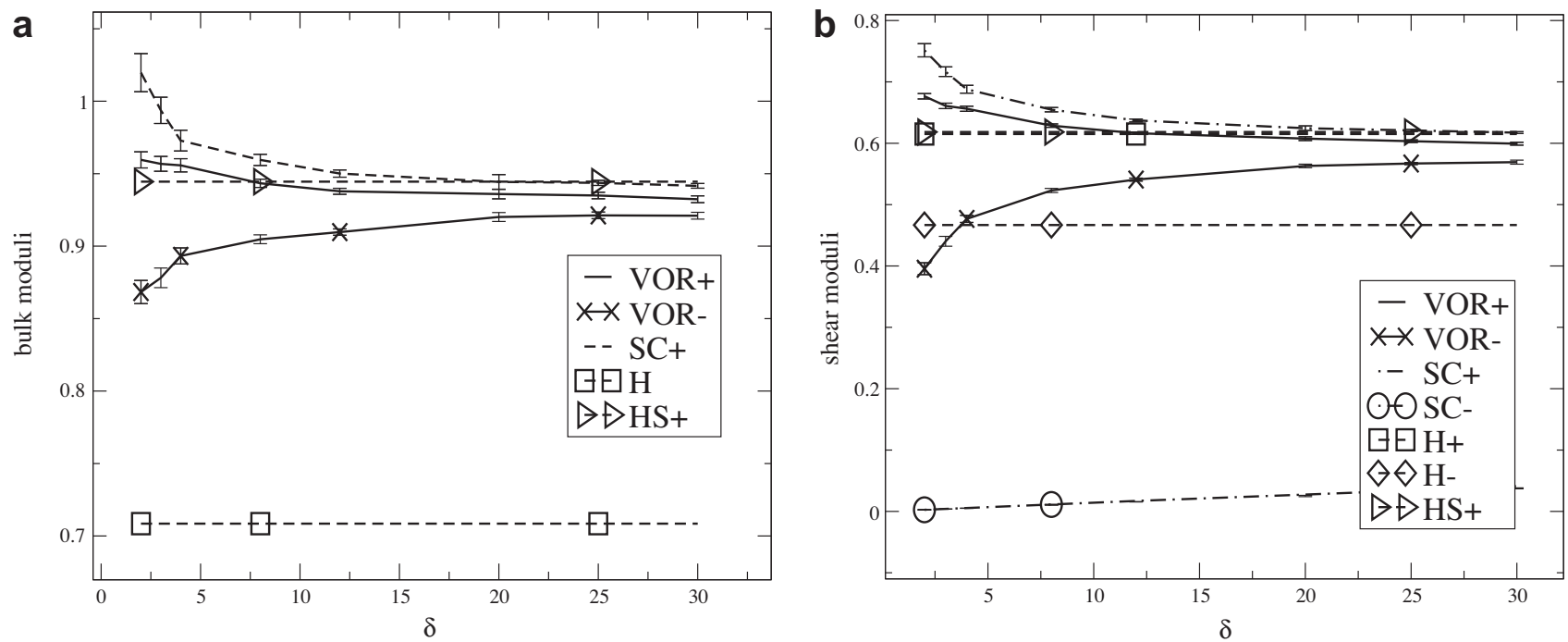

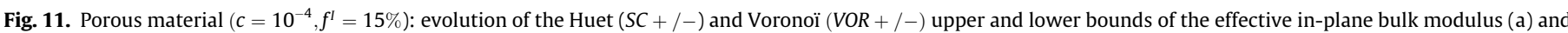
shear modulus (b) as functions of $\delta$.

randomly chosen in the large window does not satisfy the minimal distance requirement, it is rejected and a new one is generated until the distance condition is fulfilled. New disks are added until the prescribed porosity is reached. The overall inclusion volume fraction can be monitored precisely for the size of the microstructure $B(\omega)$ is much larger than the fibers diameter $d$. Since Finite Element calculations are used in this work to compute the apparent behaviors, a strictly positive minimal distance between the disks of $3.5 \% d$ (cherry pit model) has been prescribed to prevent mesh difficulties. It is well known that such a way to generate the microstructure - which is a slightly modified version of the well-known random sequential adsorption (RSA) algorithm - is not able to deal with volume fractions greater than about 35\% in 2D. To generate matrix-inclusion microstructures with higher volume fractions, it is needed to use more sophisticated algorithm such as the simulated annealing algorithm (Gusev, 1997; Rintoul and Torquato, 1997). Such an implementation is left for future work. Lastly, it should be noted that the stochastic process used to generate the microstructure of the material - i.e the modified
Poisson process sustaining the modified RSA algorithm - is unique and thoroughly characterized. Accordingly, the effective properties as well as the VOR bounds associated with this specific and completely characterized microstructure are uniquely defined and, as a consequence of Eq. (8), have the same values when $\delta \rightarrow+\infty$.

\subsection{Voronoï subdivision and non-square VEs generation}

From the large microstructure generated in Section 4.2, a Voronoï subdivision is performed by using a Matlab function. Then, similarly to Section $3.1, n$ square windows of same size $L=\delta d$ are extracted from this subdivision and are used to generate $n$ nonsquare VEs made of the set of elementary Voronoï cells the centers of which belong to a same $\delta$-sized window. The $n$ square windows of same size $\delta$ are extracted sequentially by means of a Poisson process providing the centers of the square windows. In order to prevent windows overlapping and possible statistical bias, a minimal distance of $2 \delta$ between the centers is imposed. Similarly to the implementation of the disk centers, if the current windows center 

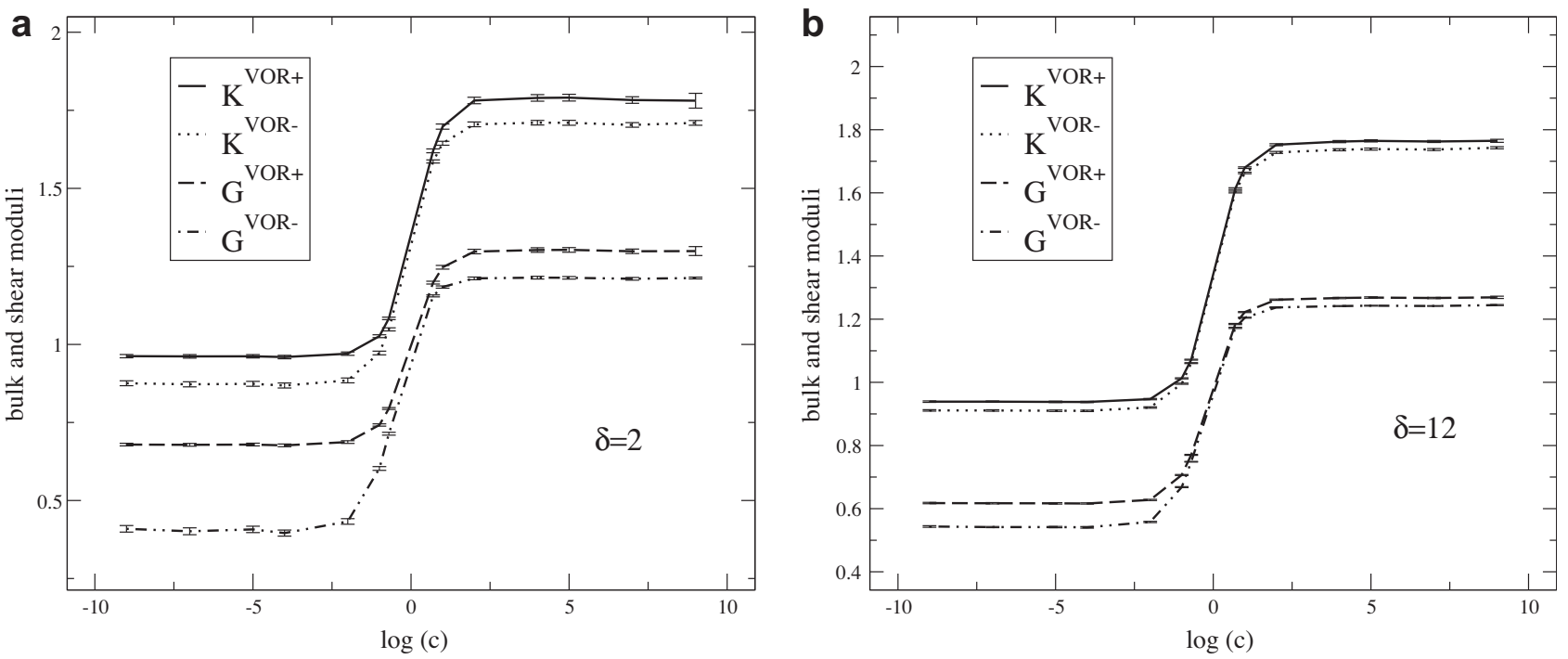

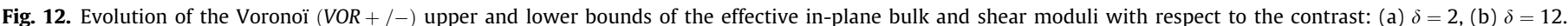
$f^{I}=15 \%$.

does not satisfy the minimal distance requirement, it is rejected and a new one is generated until the distance condition is fulfilled. Ideally, a single very large microstructure is enough to extract $n$ windows of same size $\delta$ and generate $n$ non-square VEs. However, the computational time to perform a Voronoï subdivision by Matlab seems to follow an exponential growth function of the size of the microstructure, thus leading to prohibited computational times for very large microstructures. In practice, as mentioned in the previous section, large microstructures of size $125 \delta$ are generated. For each of these microstructures, $m(\delta)$ square windows are extracted. The parameter $m(\delta)$ is adjusted numerically for each size $\delta$ once for all: for instance $m(2)=25, m(8)=5$ and for $\delta$ larger than 20 $m(\delta)=1$. The number $M(\delta)$ of generated microstructures required to built $n$ non-square VEs is given by $M(\delta)=\lfloor n / m(\delta)\rfloor+1$ where $\lfloor x\rfloor$ denotes the integer part of $x$. Such a process is performed for each size $\delta$ considered. For numerical applications, $1000 \leqslant n \leqslant 2000$. The generation of both the microstructures and the non-square VEs is performed by means of $\mathrm{C}++$ subroutines.

\subsection{Computation of the apparent behavior}

\subsection{1. local problem}

Once the non-square VEs $B_{\delta}(\omega)$ are generated, the apparent behaviors for ADBC and UTBC are computed. Since we focus on the transverse response of the composite, the local problem to be solved can be dealt with as a $2 \mathrm{D}$ problem in the plane strain framework.

In plane strain, second-order tensor fields such as the strain and in-plane components of the stress fields admit the decomposition

$\varepsilon=\varepsilon_{m} i+\varepsilon_{p s} e_{p s}+\varepsilon_{s s} e_{s s}, \quad \sigma=\sigma_{m} i+\sigma_{p s} e_{p s}+\sigma_{s s} e_{s s}$

where the second-order tensors $i, e_{p s}$ and $e_{s s}$ are given on the considered cartesian basis by

$i=e_{1} \otimes e_{1}+e_{2} \otimes e_{2}, \quad e_{p s}=e_{1} \otimes e_{1}-e_{2} \otimes e_{2}$,

$e_{s s}=e_{1} \otimes e_{2}+e_{2} \otimes e_{1}$.
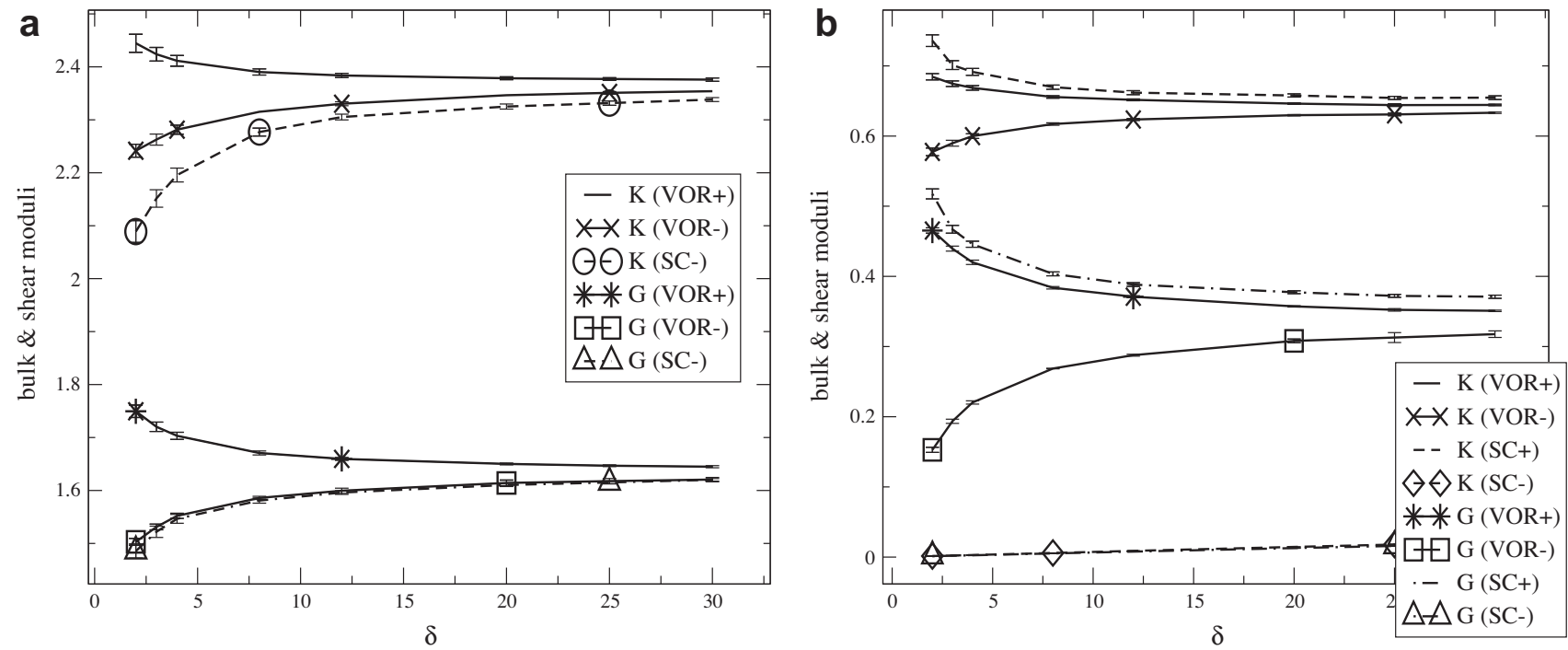

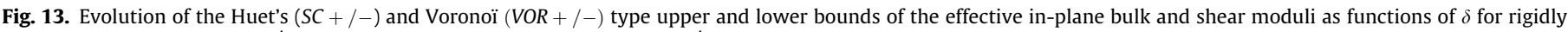
reinforced composites $\left(c=10^{4}, f^{I}=30 \%\right)$ (a) and porous materials $\left(c=10^{-4}, f^{I}=30 \%\right)(b)$. 
The scalars $\varepsilon_{m}, \varepsilon_{p s}, \varepsilon_{s s}$ (resp. $\sigma_{m}, \sigma_{p s}, \sigma_{s s}$ ) are the hydrostatic, pure shear and simple shear components of the strain (resp. stress) field. The 2D local problem to be solved is defined as

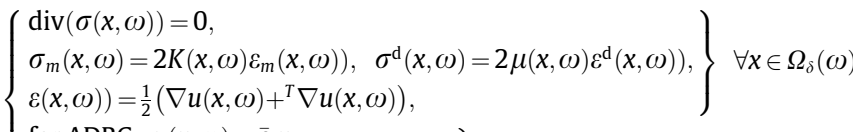

$$
\begin{aligned}
& \left.\begin{array}{l}
\text { for ADBC: } u(x, \omega)=\bar{\varepsilon} . x, \\
\text { for UTBC : } \sigma(x, \omega) \cdot n(x, \omega)=\bar{\sigma} \cdot n(x, \omega),
\end{array}\right\} \forall x \in \partial \Omega_{\delta}(\omega)
\end{aligned}
$$

where

$$
K(x, \omega)=\sum_{r=I, M} K^{r} \chi^{r}(x, \omega), K^{r}=k^{r}+\mu^{r} / 3, \mu(x, \omega)=\sum_{r=I, M} \mu^{r} \chi^{r}(x, \omega)
$$

In Eqs. (35) and (36), the superscript $d$ denotes the deviatoric part of the strain and stress tensors and $K^{\mathrm{r}}$ is the in-plane bulk modulus of phase $r$. The in-plane macroscopic strain $\bar{\varepsilon}$ and stress $\bar{\sigma}$ also take the form of Eq. (33).

\subsubsection{Apparent behavior in plane strain framework}

As explained in Section 4.1, the apparent behaviors $C_{\delta}^{d}(\omega)$ and $S_{\delta}^{t}(\omega)$ are not transversally isotropic for a VE of finite size $\delta \leqslant \delta_{\text {RVE }}$. In plane strain conditions, they are given by

$$
\begin{aligned}
& C_{\delta}^{d}(\omega)=2 K_{\delta}^{d}(\omega) J_{T}+2 \lambda_{\delta}^{d}(\omega) E_{p s}+2 \mu_{\delta}^{d}(\omega) E_{s s}+2 \alpha_{\delta}^{d}(\omega) E_{m, p s}^{s}+2 \beta_{\delta}^{d}(\omega) E_{m, s s}^{s} \\
& \quad+2 \gamma_{\delta}^{d}(\omega) E_{p s, s s}^{s} \\
& S_{\delta}^{t}(\omega)=\frac{1}{2 K_{\delta}^{t}(\omega)} J_{T}+\frac{1}{2 \lambda_{\delta}^{t}(\omega)} E_{p s}+\frac{1}{2 \mu_{\delta}^{t}(\omega)} E_{s s}+\frac{1}{2 \alpha_{\delta}^{t}(\omega)} E_{m, p s}^{s}+\frac{1}{2 \beta_{\delta}^{t}(\omega)} E_{m, s s}^{s} \\
& \quad+\frac{1}{2 \gamma_{\delta}^{t}(\omega)} E_{p s, s s}^{s},
\end{aligned}
$$

where the fourth-order tensors $J_{T}, E_{p s}, E_{s s}, E_{m, p s}^{s}, E_{m, s s}^{s}, E_{p s, s s}^{s}$ are defined by

$$
\begin{aligned}
& J_{T}=\frac{1}{2} i \otimes i, \quad E_{p s}=\frac{1}{2} e_{p s} \otimes e_{p s}, \quad E_{s s}=\frac{1}{2} e_{s s} \otimes e_{s s}, \quad E_{m, p s}^{s}=\frac{1}{2}\left(i \otimes e_{p s}+e_{p s} \otimes i\right), \\
& E_{m, s s}^{s}=\frac{1}{2}\left(i \otimes e_{s s}+e_{s s} \otimes i\right), \quad E_{p s, s s}^{s}=\frac{1}{2}\left(e_{p s} \otimes e_{s s}+e_{s s} \otimes e_{p s}\right) .
\end{aligned}
$$

The fourth-order tensor $J_{T}$ is the usual spherical projector in the transverse plane while the tensors $E_{p s}, E_{s s}$ introduced by Willot et al. (2008) are projectors on the subspace of deviatoric second-order tensors spanned by $e_{p s}$ and $e_{s s}$, respectively. The remaining tensors $E_{m, p s}^{s}, E_{m, s s}^{s}, E_{p s, s s}^{s}$ allow for the coupling between hydrostatic, pure shear and simple shear components. They are introduced to fully characterize any material anisotropy in plane strain conditions. The scalars $K_{\delta}^{d, t}(\omega)$ are the in-plane apparent bulk modulus for $\operatorname{ADBC}(d)$ and $\operatorname{UTBC}(t)$ while $\lambda_{\delta}^{d, t}(\omega), \mu_{\delta}^{d, t}(\omega)$ are the in-plane apparent pure shear and simple shear moduli, respectively. The other apparent material properties $\alpha_{\delta}^{d, t}(\omega), \beta_{\delta}^{d, t}(\omega), \gamma_{\delta}^{d, t}(\omega)$ characterize the coupling between hydrostatic, pure shear and simple shear components.

The 6 components of the apparent moduli tensor $C_{\delta}^{d}(\omega)$ are classically computed for each $\omega$-realization of this tensor by solving the local problem (35) for 3 different ADBC, i.e. $\bar{\varepsilon}=i, \bar{\varepsilon}=e_{p s}$ and $\bar{\varepsilon}=e_{s s}$, thus providing the wanted quantities by averaging the local stress over the whole VE $B_{\delta}(\omega)$ for each loading. The components of $S_{\delta}^{t}(\omega)$ are similarly derived by applying to (35) in-plane hydrostatic, pure shear and simple shear homogeneous stress components, respectively again in the plane strain framework. Any numerical scheme able to solve the local problem (35) can be employed to compute the apparent behaviors. In the sequel, the FE method will be used.

\subsection{Bounds for the effective behavior}

\subsubsection{Exact expressions}

As explained in Section 4.1, due to rotational invariance along the fiber axis, the effective behavior $C^{\text {eff }}$ and the ensemble average of the apparent behavior associated with non-square VEs, i.e. $C_{\delta}^{V O R+1-}$, are transversally isotropic. Accordingly, they read

$C^{\mathrm{eff}}=2 K^{\mathrm{eff}} J_{T}+2 G^{\mathrm{eff}} K_{T}, C_{\delta}^{\mathrm{VOR+1-}}=2 K_{\delta}^{\mathrm{VOR+1} /-} J_{T}+2 G_{\delta}^{\mathrm{VOR}+/-} K_{T}$,

where $K$ and $G$ denote the in-plane bulk and shear moduli. The tensor $K_{T}$, introduced first by Walpole (1981) and defined by $K_{T}=E_{p s}+E_{s s}=I-J_{T}$, is the usual projector on the subspace of purely in-plane deviatoric second-order tensors and $I$ is the fourth-order symmetric identity tensor. Recalling the definition (29) of $C_{\delta}^{V O R+/-}$ together with Eqs. (37) and applying purely hydrostatic or deviatoric macroscopic strain to Eq. (22) provide the expected new upper and lower bounds of the effective in-plane bulk and shear moduli of the composite as

$$
\begin{aligned}
& \frac{1}{2} J_{T}::\left(E\left(S_{\delta}^{t}\right)\right)^{-1}=K_{\delta}^{V O R-} \leqslant K^{\mathrm{eff}} \leqslant K_{\delta}^{V O R+}=\frac{1}{2} J_{T}:: E\left(C_{\delta}^{d}\right), \\
& \frac{1}{4} K_{T}::\left(E\left(S_{\delta}^{t}\right)\right)^{-1}=G_{\delta}^{V O R-} \leqslant G^{\mathrm{eff}} \leqslant G_{\delta}^{V O R+}=\frac{1}{4} K_{T}:: E\left(C_{\delta}^{d}\right) .
\end{aligned}
$$

As mentioned in Section 3.3, the expectations in Eq. (40) and in all the formulae associated with the Voronoï type bounds should be understood in the sense of Eq. (32). In numerical applications, the computation of the in-plane bounds of the effective behavior are carried out by means of formula (40). In practice, it amounts to compute $E\left(C_{\delta}^{d}\right),\left(E\left(S_{\delta}^{t}\right)\right)^{-1}$ and to extract, on the one hand, the $J_{T}$ components of these tensors which correspond to the bounds of the effective in-plane bulk modulus and, on the other hand, the half sum of their $E_{p s}$ and $E_{s s}$ components to derive the bounds of the effective in-plane shear modulus. However, for transversally isotropic behaviors as considered in this study, the Voronoï type bounds can also be estimated by the following expressions

$$
\begin{aligned}
& K_{\delta}^{V O R+}=E\left(K_{\delta}^{d}\right), K_{\delta}^{V O R-}=\left(E\left(\frac{1}{K_{\delta}^{t}}\right)\right)^{-1}, G_{\delta}^{V O R+}=E\left(\lambda_{\delta}^{d}\right)=E\left(\mu_{\delta}^{d}\right)=E\left(\frac{\lambda_{\delta}^{d}+\mu_{\delta}^{d}}{2}\right), \\
& G_{\delta}^{V O R-}=\left(E\left(\frac{1}{\lambda_{\delta}^{t}}\right)\right)^{-1}=\left(E\left(\frac{1}{\mu_{\delta}^{t}}\right)\right)^{-1}=\frac{1}{2}\left(\left(E\left(\frac{1}{\lambda_{\delta}^{t}}\right)\right)^{-1}+\left(E\left(\frac{1}{\mu_{\delta}^{t}}\right)\right)^{-1}\right) .
\end{aligned}
$$

Formulae (41) are easily derived by making use of both the transverse isotropy and definition of $C_{\delta}^{V O R+/-}$.

\subsubsection{Approximate expressions}

The evaluation of the bounds (40) requires to compute the expectations $E\left(C_{\delta}^{d}\right)$ and $\left(E\left(S_{\delta}^{t}\right)\right)^{-1}$. In practice, only estimates of the exact value of these ensemble averages are computed according to definition (30). However, the obtained results are not strictly bounds of the effective behavior. They are only numerical approximations of the exact bounds and their accuracy increases with the number of realizations $n$. The numerical accuracy of these approximations can be quantified by means of the notion of confidence interval (CI). If we consider a random variable $X(\omega)$ and the unbiased estimators $\hat{\bar{X}}(\omega)=\frac{1}{n} \sum_{i=1}^{n} X_{i}(\omega), \widehat{S}_{n}^{2}=\frac{1}{n-1} \sum_{i=1}^{n}\left(X_{i}(\omega)-\hat{\bar{X}}_{n}(\omega)\right)^{2}$ of its mean $E(X)$ and variance $\sigma^{2}(x)$, where $X_{1}(\omega), \ldots, X_{n}(\omega)$ are $n$ independent replications of $X(\omega)$, the confidence interval of level $1-\alpha \in] 0,1[$ of $E(X)$ associated with the random $n$-sample $\left(X_{1}(\omega), \ldots, X_{n}(\omega)\right)$ is defined as a random interval $\hat{C}_{1-\alpha}^{n}(E(X))(\omega)$ of the real line such that $P\left(\hat{C} I_{1-\alpha}^{n}(E(X))(\omega) \ni E(X)\right)=1-\alpha$. In the symmetric case such an interval is of the form: $\hat{C I}_{1-\alpha}^{n}(E(X))$ $(\omega)=\left[\hat{\bar{X}}_{n}(\omega)-t(\alpha, n) \frac{\widehat{S}_{n}(\omega)}{\sqrt{n}}, \hat{\bar{X}}_{n}(\omega)+t(\alpha, n) \frac{\widehat{S}_{n}(\omega)}{\sqrt{n}}\right]$ where $t(\alpha, n)$ is a 
positive real number whose value is estimated from the equality $P\left(\frac{\left|\hat{\bar{X}}_{n}(\omega)-E(X)\right|}{\widehat{S}_{n}(\omega) / \sqrt{n}} \leqslant t(\alpha, n)\right)=1-\alpha$ and by using the fact that the random variable $\sqrt{n}\left(\hat{\bar{X}}_{n}(\omega)-E(X)\right) / \widehat{S}_{n}(\omega)$ approximately follows a Student distribution with $n-1$ degrees of freedom if $n$ is sufficiently large. From this result, a deterministic $(1-\alpha)$-confidence interval $C I_{1-\alpha}^{n}(E(X))$ can be constructed for $E(X)$. Let $\left(X_{1}(\omega), \ldots, X_{n}(\omega)\right)$ be a numerical n-sample of $X(\omega)$, where $\left(X\left(\omega_{i}\right), \omega_{i} \in \Theta, 1 \leqslant i \leqslant n\right)$ are $n$ independent realizations of $X(\omega)$. Then, $C_{1-\alpha}^{n}(E(X))$ is given by

$C I_{1-\alpha}^{n}(E(X))=\left[\bar{X}_{n}-t(\alpha, n) \frac{S_{n}}{\sqrt{n}}, \bar{X}_{n}+t(\alpha, n) \frac{S_{n}}{\sqrt{n}}\right]$

where $\bar{X}_{n}=\frac{1}{n} \sum_{i=1}^{n} X\left(\omega_{i}\right)$ and $S_{n}^{2}=\frac{1}{n-1} \sum_{i=1}^{n}\left(X\left(\omega_{i}\right)-\bar{X}_{n}\right)^{2}$. Such an interval can be seen as a particular realization of the random interval $\hat{C} I_{1-\alpha}^{n}(E(X))(\omega)$ (Casella and Berger, 2001). Thus, the length $\frac{t(\alpha, n) S_{n}}{\sqrt{n}}$ is a probabilistic characterization of the relative accuracy of the estimated expectation value $\bar{X}_{n}$. The result (42) is theoretically established only in the case of unbiased estimates $\bar{X}_{n}$ and $S_{n}^{2}$ which are defined by giving the same weight to each realization. In the numerical applications, we consider an extension of (42) where $\bar{X}_{n}$ and $S_{n}$ are now defined by means of different weight $V_{\delta}\left(\omega_{i}\right) / \bar{V}_{\delta}$ as

$\bar{X}_{n}=\frac{1}{n} \sum_{i=1}^{n} \frac{V_{\delta}\left(\omega_{i}\right)}{\bar{V}_{\delta}} X\left(\omega_{i}\right), S_{n}^{2}=\frac{1}{n-1} \sum_{i=1}^{n} \frac{V_{\delta}\left(\omega_{i}\right)}{\bar{V}_{\delta}}\left(X\left(\omega_{i}\right)-\bar{X}_{n}\right)^{2}$.

where $\bar{V}_{\delta}=\frac{1}{n} \sum_{i=1}^{n} V_{\delta}\left(\omega_{i}\right)$.

It should be noted that the $\mathrm{CI}(42)$ associated with definitions (43) has no rigorous theoretical foundation and is only a heuristic extension of the classical definition (42) associated with equalweighted ensemble average.

An interesting illustration of the $\mathrm{CI}$ notion can be obtained by introducing the estimated upper $K_{\delta, \text { est }}^{+}$and lower $K_{\delta, \text { est }}^{-}$bounds of the effective behavior. These bounds are defined by

$K_{\delta, e s t}^{+}=\overline{K_{\delta}^{d}}+\ell^{C I}\left(E\left(K_{\delta}^{d}\right)\right), \quad K_{\delta, e s t}^{-}=\left(\overline{1 / K_{\delta}^{t}}+\ell^{C I}\left(1 / K_{\delta}^{t}\right)\right)^{-1}$

where $\ell^{C I}(E(X))$ denotes the half length of the $(1-\alpha)$-confidence interval of $E(X)$, i.e. $\ell^{C I}(E(X))=\frac{t(\alpha, n) S_{n}}{\sqrt{n}}$. To simplify the notations in Eq. (44), the dependence on $n$ of $\bar{X}$ and on $n$ and $\alpha$ of both $\ell^{C I}(E(X))$ and $X_{\text {est }}^{ \pm}$has been omitted. The definition of the confidence interval implies

$P\left(K_{\delta, e s t}^{-} \leqslant K^{\text {eff }} \leqslant K_{\delta, \text { est }}^{+}\right) \geqslant 1-\alpha$.

Note that the ensemble averages $\bar{X}$ used in Eq. (44) should be understood as weighted ensemble averages as defined in (43) when $K_{\delta \text {,est }}^{+}$and $K_{\delta, \text { est }}^{-}$are derived by means of the Voronoï type approach whereas the same notation $\bar{X}$ corresponds to the classical equalweighted ensemble average when associated with the classical Huet's approach. The same holds true for the estimate $S$ of the standard deviation.

\subsubsection{Specificities associated with square VEs}

For square VEs, the ensemble averages of the apparent behavior, i.e. $C_{\delta}^{S C+/-}$, exhibit quadratic symmetry unlike the bounds $C^{V O R+1-}$ which are (almost) transversally isotropic. Indeed, although we still have rotational invariance along the fiber axis $e_{3}$ of both the constitutive relations of the phases and the phase distribution (when averaging over the whole set of realizations), the shape of each square VE whose sides are parallel to the reference axes $e_{1}, e_{2}$ leads to a quadratic symmetry with respect to $e_{1}, e_{2}$. Therefore,

$C_{\delta}^{S C+/-}=2 K_{\delta}^{S C+/-} J_{T}+2 \lambda_{\delta}^{S C+/-} E_{p s}+2 \mu_{\delta}^{S C+/-} E_{S S}$.

Applying the definition (29) of the Huet's bounds to (37) and comparing the obtained result to expression (46) provide the following expression for the in-plane bulk $K_{\delta}^{S C+/-}$, pure shear $\lambda_{\delta}^{S C+/-}$ and simple shear $\mu_{\delta}^{\mathrm{SC}+/-}$ moduli

$$
\begin{aligned}
& K_{\delta}^{S C+}=E\left(K_{\delta}^{d}\right)=\frac{1}{2} J_{T}:: C_{\delta}^{S C+}, K_{\delta}^{S C-}=\left(E\left(1 / K_{\delta}^{t}\right)\right)^{-1}=\frac{1}{2} J_{T}::\left(S_{\delta}^{S C-}\right)^{-1}, \\
& \lambda_{\delta}^{S C+}=E\left(\lambda_{\delta}^{d}\right)=\frac{1}{2} E_{p s}:: C_{\delta}^{S C+}, \quad \lambda_{\delta}^{S C-}=\left(E\left(1 / \lambda_{\delta}^{t}\right)\right)^{-1}=\frac{1}{2} E_{p s}::\left(S_{\delta}^{S C-}\right)^{-1}, \\
& \mu_{\delta}^{S C+}=E\left(\mu_{\delta}^{d}\right)=\frac{1}{2} E_{p s}:: C_{\delta}^{S C+}, \mu_{\delta}^{S C-}=\left(E\left(1 / \mu_{\delta}^{t}\right)\right)^{-1}=\frac{1}{2} E_{p s}::\left(S_{\delta}^{S C-}\right)^{-1} .
\end{aligned}
$$

In Eq. (47) as well as in all the expressions associated with the Huet's SC type bounds, the expectations should be understood in a classical sense, i.e. with a same weight ascribed to each realization.

Applying pure shear $\left(e_{p s}\right)$ or simple shear $\left(e_{s s}\right)$ macroscopic strain $\bar{\varepsilon}$ and stress $\bar{\sigma}$ loadings to Eq. (14) and making use of relations (47), the following bounds for the in-plane effective shear modulus are obtained

$\sup \left(\lambda_{\delta}^{S C-}, \mu_{\delta}^{S C-}\right) \leqslant G^{\text {eff }} \leqslant \inf \left(\lambda_{\delta}^{S C+}, \mu_{\delta}^{S C+}\right)$.

To the best of the authors knowledge, the bounding property (48) which makes use of the quadratic anisotropy of $C_{\delta}^{S C+1-}$ has never been used nor noticed before. Indeed, the Huet's upper and lower bounds have been until now considered to be isotropic in the literature. This assumption of isotropy was supported by various numerical simulations performed for some range of parameters such as for instance sufficiently large VE $\delta$ size or finite contrast. Although we find again in this study that Huet's bounds are isotropic for some simulations - e.g. for reinforced composites, the lower bound is nearly isotropic (up to $1 \%$ of accuracy) - their quadratic anisotropy Eq. (46) which is theoretically established at the beginning of this section by symmetry arguments is well confirmed by other numerical simulations. For instance, for rigidly reinforced composites the numerical estimate of the Huet's upper bound evaluated for a inclusion volume fraction $f^{I}=15 \%$ - provides a quadratic behavior $\left(\lambda_{\delta}^{S C+} \neq \mu_{\delta}^{S C+}\right)$ as illustrated in Fig. 8 which reports the evolution of the Huet's in-plane shear moduli upper bounds $\lambda_{\delta}^{S C+}, \mu_{\delta}^{S C+}$ as functions of $\delta$.

At last, the evolution of Huet's SC shear upper bounds can be predicted by a simple approach inspired from the one presented in Section 2.3 and applied to the in-plane bulk modulus. When this latter approach (Eq. (19)) is also applied to the in-plane pure-shear modulus, thus leading to $\lambda_{\delta}^{A b}=\lambda^{\text {eff }}+\lambda^{(2)}\left(1 / \delta-1 / \delta^{2}\right)$, it is still observed a good agreement between the approximate closed-form bound $\lambda_{\delta}^{A b}$ and the Huet's upper bound $\lambda_{\delta}^{S C+}$ as shown on Fig. 8. However, when applied to the in-plane simple-shear modulus $\mu_{\delta}^{A b}=\mu^{\text {eff }}+\mu^{(2)}\left(1 / \delta-1 / \delta^{2}\right)$, the decrease of $\mu_{\delta}^{A b}$ mainly in inverse proportion of $\delta$ does not reproduce the evolution of Huet's SC upper bound $\mu_{\delta}^{S C+}$. The reason lies in the fact that the simple-shear uniform strain loading is parallel to the boundaries of the square cell. For such a loading, the nearly rigid particles which intersect the edges of the cell are no longer strained but in fact rotate inside the matrix unlike the case of pure-shear uniform strain solicitations which enforce actual deformations to the boundary particles. Therefore, these particles do not generate the blow-up of the strain energy observed for $\mu_{\delta}^{S C+}$ when simple-shear uniform strain are applied. However, there is one exception to this situation: the particles which intersect one of the four corners of a square VE - more precisely the particles which intersect two different edges - are always deformed even when submitted to simple shear loading. Although not many, these at most 4 nearly rigid particles can explain the blow-up of the strain energy under simple shear deformation. To see if such a conjecture holds true, we make again the reasoning on which relies on the simple approach presented in Section 2.3. The part of the strain energy due to the particles which 
intersect the cell corners is taken into account by means of a new subdivision of the square VE in two parts. The first part $B_{\delta}^{(1)}(\omega)$ of volume $V_{\delta}^{(1)}=4 d^{2}$ consists of the area associated with the 4 corners such that all the particles which intersect the cell corners belong to this area. The second part $B_{\delta}^{(2)}(\omega)$ of volume $V_{\delta}^{(2)}=L^{2}-V_{\delta}^{(1)}$ is the core of the $\mathrm{VE}$, i.e. the part inside which the nearly rigid particles are not enforced to be strained by ADBC in simple shear (see Fig. 5). From this subdivision, the ratio $V_{\delta}^{(1)} / V_{\delta}$ is now proportional to $1 / \delta^{2}$ instead of $\left(1 / \delta-1 / \delta^{2}\right)$ as it was the case for the simple model associated with a pure-shear uniform strain. By reproducing the reasoning introduced in Section 2.3, we get the following closed-form approximate bound for the effective shear modulus

$\mu^{\mathrm{eff}} \leqslant \mu_{\delta}^{\mathrm{Ab}}=\mu^{\mathrm{eff}}+\frac{\mu^{(2)}}{\delta^{2}}$.

As shown in Fig. 8, the agreement between the approximate and Huet's bounds after a fitting of the constant $\mu^{(2)}$ at $\delta=30$ is quite good. This shows that the blow-up of the strain energy under simple shear uniform strain solicitations is due to the predominant contribution of the nearly rigid particles intersecting the cell corners to the apparent behavior.

\subsection{Numerical implementation}

For both the Voronoï and Square Cells approach, the apparent behaviors are computed by making use of the FE software CAST3M (CEA, 2003) according to the procedure presented in Section 4.4. However, the meshing is performed differently according to the shape (square or non-square) of the VEs.

\subsubsection{Proposed approach}

For the Voronoï approach, a meshing procedure based on the following principles has been defined. First, we make use of the Voronoï subdivision on which rely the non-square VEs introduced in Section 3.1. All Voronoï cells of a given non-square VE are individually meshed and then gathered together to rebuilt the whole VE, following a methodology very similar to the one introduced by Danielsson et al. (2007). The meshing of each Voronoï cell is easily performed by making use of the standard meshing command of CAST3M. The way to mesh a Voronoï cell mainly relies on the following considerations. The first one aims at providing accurate estimates of the local fields even in areas where they are subjected to strong gradients, especially the ones located between close inclusions. This can be easily realized by partitioning each Voronoï cell into isosceles triangular subcells the apices of which consist of both the center and two adjacent apices of the Voronoï cell. For the whole Voronoï cells, we ascribe to the matrix part of each Voronoï subcell the same finite element discretization number along the radial directions of its inclusion. Such a procedure leads to fine (resp. crude) radial meshes inside the matrix phase for each triangular Voronoï subcell the inclusion of which is close (resp. far) to its outer boundary. Note that the fact for an inclusion of a Voronoï cell to be close (resp. far) from one segment of its outer boundary amounts to being close (resp. far) from the inclusion of the adjacent Voronoï cell sharing the same segment as boundary. The second consideration is based on the Eshelby solution of a heterogeneous inclusion inside an infinite matrix, both phases being linear elastic (Eshelby, 1957). The local strain associated with this problem is heterogeneous in the matrix. Its spatial variations although quite significant near the matrix-inclusion interface decrease quickly in the radial direction of the inclusion and the strain field becomes homogeneous far enough of the inclusion. In view of the radial decrease of the local strain solution of the Eshelby problem inside the matrix, we build a mesh whose ascribed densities vary in inverse proportion to the distance from the borders of the inclusions. Similarly, as the strain is homogeneous inside the inclusion of the Eshelby problem, the density of the mesh is reduced in the center of the inclusions. Finally, in order to make compatible the meshes of two adjacent cells, the discretization along a segment of a Voronoï cell relies on a number of elements which depends only on the length of this segment and the distance to the center of the cell, which is the same for adjacent cells. A detailed explanation of the meshing algorithm is given in Salmi (2011). Fig. 6(a) provides an example of obtained mesh for a non-square VE consisting of two Voronoï cells.

In order to quantify the numerical errors generated by the proposed numerical scheme, we studied the influence of the number of finite elements on the apparent behaviors associated with ADBC and UTBC. The numerical study is performed on a non-square VE of size $\delta=12$ comprised of 27 rigid inclusions $\left(c=10^{4}\right)$ in proportion $f^{I}=15 \%$ and submitted to ADBC or UTBC, respectively. Eightnoded quadratic elements are used to carry out the calculation. The results are depicted in Fig. 6(b) which reports the evolution of the relative accuracy $X_{\delta}^{\text {rela }}\left(n_{e}\right)=\left(X_{\delta}\left(n_{e}\right)-X_{\delta}\left(n_{e}^{\infty}\right)\right) / X_{\delta}\left(n_{e}^{\infty}\right)$ as a function of the number of Finite Element $n_{e}$ where $X_{\delta}$ accounts for the different apparent moduli $K_{\delta}^{d, t}, \mu_{\delta}^{d, t}, \lambda_{\delta}^{d, t}$. The relative accuracies reported on Fig. 6(b) are computed for $n_{e}^{\infty}=25000$ since it has been numerically noticed that these moduli do not evolve any longer beyond. In the following, all the computations are carried out with a mesh size corresponding to a relative precision of $0.1 \%$.

\subsubsection{Classical approach}

For the classical Square Cell approach, the geometries of the VEs are more complex because of the inclusions intersecting the VE boundaries. The standard routines in CAST3M turn out not to be appropriate to generate such meshes. That's why the specialized mesh generator Netgen (Schoberl et al., 2003) specifically designed for random microstructures is preferred and the obtained meshes are exported to CAST3M to compute the apparent behaviors with the same procedures as for the new bounds. It should however be noticed that this generic mesh procedure is not optimized with respect to the particular microstructure under consideration. It generates meshes with almost uniform densities, and, as a consequence, much larger numbers of elements for a given accuracy. This is illustrated by the results depicted in Fig. 6(c) which gives the evolution of the relative accuracy for a square VE, again of size $\delta=12$ and made of rigid inclusions in proportion $f^{I}=15 \%$ for ADBC or UTBC, respectively. The results are now computed with $n_{e}^{\infty}=80000$. One can observe that to reach a relative accuracy of $0.1 \%$ with Netgen requires to use five times more elements than with the Voronoï-type mesh. This shows that the new bounding procedure is not only more efficient from a theoretical point of view, but also from a numerical one as it allows to generate meshes in a much easier and more efficient way.

\section{Results}

Numerical applications have been run for several contrasts $\left(c=10^{4}, 10^{2}, 5,10^{-4}\right)$ and volume fractions $\left(f^{I}=15 \%, 30 \%\right)$ thus taking into account three different types of random matrix-inclusion composites: rigidly reinforced composites $\left(c=10^{4}\right)$, twophase materials $\left(c=5,10^{2}\right)$ and porous materials $\left(c=10^{-4}\right)$. For all numerical applications, $n=2000$ for $2 \leqslant \delta<20$ or $n=1000$ for $20 \leqslant \delta \leqslant 30$. A 0.99 -confidence interval (i.e. $\alpha=0.01$ ) is used for the mean $E(X)$. For such an interval, the Student parameter $t(\alpha, n)$ satisfies $t(0.01, n)=2.56$ for all $n \geqslant 100$.

The variations with respect to $\delta$ of the Voronoï and Huet's SC bounds for the effective shear modulus of a rigidly reinforced composite $\left(f^{I}=15 \%\right)$ are depicted in Fig. 7 . For all the curves reported in this paper, the error bars refer to the symmetric confidence inter- 
val of the considered expectation. As shown in Fig. 7(a), the Voronoï type upper bound $G_{\delta}^{V O R+}$ improves on both the Huet's upper bounds $\lambda_{\delta}^{S C+}$ and $\mu_{\delta}^{S C+}$ very significantly - up to $7000 \%$ for small values of $\delta$. The Voronoï lower bound also slightly improves on the Huet's lower bounds (see Fig. 7(b)). Furthermore, the upper and lower Voronoï type bounds of $G^{\text {eff }}$ are quite sharp. They quickly converge toward the effective shear modulus when $\delta$ increases. For instance, for $\delta \geqslant 20$ the relative gap between both bounds $\left(\Delta G_{\delta} / G_{\delta}\right)^{V O R}=$ $\left(G_{\delta}^{\mathrm{VOR}+}-G_{\delta}^{\mathrm{VOR}-}\right) /\left(G_{\delta}^{\mathrm{VOR}+}+G_{\delta}^{\mathrm{VOR}-}\right) \quad$ satisfies $\quad\left(\Delta G_{\delta} / G_{\delta}\right)^{\mathrm{VOR}} \leqslant 0,92 \%$. For the rigidly reinforced composite, it should be noted that the pure-shear and simple-shear SC lower bounds are the same unlike their corresponding upper bounds which exhibit a pronounced quadratic anisotropy.

The classical Hashin and Shtrikman (1963) lower bound (HS-) relative to fiber reinforced composites (Willis, 1977) as well as the Hashin and Rosen (1964) upper $\left(\mathrm{H}^{+}\right)$and lower $(\mathrm{H}-)$ bounds are also reported on Fig. 7(b) for comparison purpose. Although the Hashin and Shtrikman lower bound as well as the Hashin upper bound provide very accurate estimates of the effective behavior, the Voronoï type bounds yield better predictions for $\delta \geqslant 25$. It should be noted that the Hashin bounds are not bounds for the microstructures considered in this work unlike the Hashin and Shtrikman lower bound. Indeed, matrix-fiber composites with identical cylindrical fibers randomly and isotropically distributed in the transverse plan are not exactly Composite Cylinders Assemblages (CCA) for which the Hashin and Rosen bounds rigorously apply. However, since their microstructures have a significant degree of similarity with a CCA, the Hashin and Rosen bounds provide useful information about the effective behavior of the matrix-fiber composites considered in this study. This remark explains why the Hashin upper bound - which is only an estimate of the effective behavior for the microstructures considered in this paper - violate the Voronoï lower bound. But it also explains why the Hashin bounds provide relatively good estimates of the effective moduli (Fig. 7(b)). These results are also observed for the other contrasts.

Thereby, as shown on Fig. 7, the artificial over-estimation induced by a direct application of uniform boundary conditions on nearly rigid particles can be almost fully removed by the Voronoï type approach. The estimates derived by this new approach provide accurate predictions of the effective behavior even for relatively small values of $\delta$. Furthermore, they retains their bound status associated with uniform BC unlike the estimates of the effective behavior derived by extending Huet's approach to periodic BC (e.g. Kanit et al., 2003; Ostoja-Starzewski, 2006) which, although efficient, are not bounds.

Numerical simulations have also been performed for the inplane bulk modulus. As expected, the same trends are observed.

In what follows, although Huet's SC bounds exhibit a quadratic symmetry we choose to only report their isotropic projection $K_{\delta}^{S C+1-}, G_{\delta}^{S C+/-}$ for the sake of simplicity. However, it should be noted that all the results of the comparisons between the isotropic Huet's and Voronoï type bounds presented hereafter still hold when applied to $\lambda_{\delta}^{S C+1-}$ and to $\mu_{\delta}^{S C+/-}$ instead of $G_{\delta}^{S C+/-}$. The in-plane isotropic moduli are defined by

$$
\begin{aligned}
& G_{\delta}^{S C+}=\frac{1}{2} K_{T}:: C_{\delta}^{S C+}=\frac{1}{2}\left(\lambda_{\delta}^{S C+}+\mu_{\delta}^{S C+}\right), \\
& G_{\delta}^{S C-}=\frac{1}{2} K_{T}::\left(S_{\delta}^{S C-}\right)^{-1}=\frac{1}{2}\left(\lambda_{\delta}^{S C-}+\mu_{\delta}^{S C-}\right),
\end{aligned}
$$

while $K_{\delta}^{S C+/-}$ are still given by $(47)_{1,2}$. Of course, since $\lambda_{\delta}^{S C+/-}$ and $\mu_{\delta}^{S C+/-}$ are upper and lower bounds of the effective shear modulus $G^{\text {eff }}$, this property still holds for $G_{\delta}^{S C+/-}$.

The evolutions with respect to $\delta$ of the estimated upper $K_{\delta, \text { est }}^{+}$ and lower $K_{\delta \text { est }}^{-}$bounds defined in (44) are reported in Fig. 9 for a two-phase material with high contrast $\left(c=10^{2}, f^{I}=15 \%\right)$. As mentioned in Section 4.5.2, the interest to make use of the estimated bounds $K_{\delta, e s t}^{+}, K_{\delta, \text { est }}^{-}$instead of their approximated values $\overline{K_{\delta}^{d}},\left(\overline{1 / K_{\delta}^{t}}\right)^{-1}$ is their rigourous interpretation in terms of probability as described by Eq. (45) for $\alpha=0.01$. In Fig. 9, the same trends as in the case of the rigidly reinforced composite can be observed. Furthermore, it should be noted that the Voronoï type upper and lower bounds associated with both the reinforced composite and the two-phase material with high contrast $\left(c=10^{2}\right)$ provide the same numerical results unlike the Huet's upper bound which, in addition to being too stiff, exhibits a strong non-physical dependence with the moduli of the fibers.

The variation of the Huet's and Voronoï type bounds of the inplane bulk and shear effective moduli with respect to $\delta$ are depicted in Figs. 10(a) and (b), respectively, for a two-phase material with medium contrast $\left(c=5, f^{l}=15 \%\right)$. Again, Fig. 10 shows that the Voronoï's type bounds improve both the upper and lower Huet's bounds which, as expected for such a low contrast, provides reasonably good predictions of the effective properties even for relatively small $\delta$.

We also investigated the apparent behavior of porous materials $\left(c=10^{-4}\right)$. The evolutions of the SC and VOR bounds of either the in-plane bulk or shear effective moduli with respect to $\delta$ are depicted in Fig. 11 for $f^{I}=15 \%$. Similarly to reinforced composites, the Voronoï type bounds improve on both the Huet's SC bounds of the inplane bulk and shear moduli: the lower one (which is not reported in Fig. 11(a) because it is too low) as expected but also slightly the upper one. Although the VOR bounds of $K^{\text {eff }}$ and $G^{\text {eff }}$ are still quite sharp, they converge toward the effective properties slower than the ones obtained for the rigidly reinforced composite. Conversely, they improve on the Hashin and Shtrikman bound for values of $\delta$ much smaller $(\delta \geqslant 12)$ than the ones required for the rigidly reinforced composite $(\delta \geqslant 25)$. In order to check that the VOR bounds of both the so-called rigidly reinforced composite $\left(c=10^{4}\right)$ and porous material $\left(c=10^{-4}\right)$ respectively correspond to the ones of a real rigidly reinforced composite $(c=+\infty)$ and porous material $(c=0)$, we report on Fig. 12 the evolutions of the VOR upper and lower bounds of the effective in-plane bulk and shear moduli as functions of the contrast - with values ranging from $10^{-9}$ to $10^{9}$. Two different values of the VE size $(\delta=2,12)$ are considered. As seen in Fig. 12, the VOR bounds of the effective bulk and shear moduli do not evolve any more - up to the numerical accuracy - when the contrast is less than $10^{-3}$ or larger than $10^{2}$, thus showing that each VOR bound reaches a limit which corresponds to situation of infinite contrasts. Furthermore, the values of these limits are attained for both the so-called rigidly reinforced composites $\left(c=10^{4}\right)$ or porous materials $\left(c=10^{-4}\right)$ considered in this study.

Lastly, we investigated the influence of the inclusion volume fraction. For that, we report the evolutions of the SC and VOR bounds of the effective in-plane bulk and shear moduli with respect to $\delta$ for both a rigidly reinforced composite (Fig. 13 and a porous material Fig. 13(b)) for $f^{I}=30 \%$. The trends are similar to those observed for $f^{I}=15 \%$ except that now the convergence toward the effective moduli occurs for larger $\delta$. For instance, if we consider the evolution of the in-plane shear modulus bounds of the porous material, which is the most disadvantageous situation, the relative gap between the upper and lower VOR bounds $\left(\Delta G_{\delta} / G_{\delta}\right)^{V O R}$ at $\delta=30$ worths $4.5 \%$ for $f^{I}=30 \%$ instead of $2.5 \%$ for $f^{I}=15 \%$. This result can be explained by the fact that the more the pores (or particles) are close, the more their mechanical interactions increase and therefore the more the fluctuations of the apparent properties grow. Such an enhancement of the apparent behavior fluctuations can induce an increase of the RVE size. However, this explanation may not hold for all pore or particle volume fractions. Indeed, when $f^{I}$ tends to large values, e.g. the percolation limit, the random distribution of the inclusions is geometrically 
more constrained and therefore becomes more homogeneous. Although the mechanical interactions are reinforced through the enhancement of the volume fraction, the lessening of the microstructure heterogeneity for large inclusion volume fractions may or may not induce a decreasing of the fluctuations of the apparent properties depending on which of both above-mentioned effects dominates. At last, other calculations performed for different volume fractions $10 \%, 20 \%, 25 \%$ for rigidly reinforced composites or porous materials have showed that the VOR bounds always significantly improve on the Huet's bounds. However, to gain more insight about the influence of the particle or pore volume fraction on the apparent behavior and its fluctuations would require to perform a statistical analysis of the apparent properties for several volume fractions ranging from the dilute limit up to the percolation threshold. Such a study is out of the scope of this paper.

\section{Conclusion}

A new micro-mechanical approach relying on ensemble averages, in an appropriate sense, of the apparent behaviors associated with non-square (or non-cubic) VEs submitted to ADBC or UTBC has been developed. This method leads to new rigorous bounds for the effective behavior of linear matrix-inclusion random composites and enables to get rid of the limitation of Huet's approach for large contrast, i.e. the blow up or vanishing of the SC bounds of the effective properties due to direct applications of uniform $\mathrm{BC}$ to the particles. As a first illustration this method has been applied to a two-phase composite consisting of a matrix and aligned identical cylindrical fibers randomly and isotropically distributed in the transverse plane, each phase following Hooke's law. The new VOR bounds of the effective transverse properties derived by this approach are quite sharp and converge quickly to the effective properties, for all contrasts, even infinite. They improve on the classical Huet's bounds for all contrasts, the large ones as expected but also the small ones, and for all particle volume fractions. They can also be obtained at a lower numerical cost.

The applications have intentionally been limited to the transverse properties of the simplest three-dimensional microstructure for which this approach applies. They can be extended to full 3D loadings and to more general 3D microstructures, with anisotropic phases and phase distributions. They are however restricted to composites with a matrix/inclusion-type microstructure, with separated inclusions, making it possible to design VEs avoiding any direct application of boundary conditions to the inclusions. For instance, one could deal with inclusions of different shapes (ellipsoidal particles or short fibers with random orientation, etc.) and random size. For that, it would be necessary to subdivise the RVE with a more general scheme than the classical point-based Voronoï procedure by making use for instance of the distance function as explained in Section 3.1. Such extensions would require to design appropriate meshing procedures associated with the subdivision relying on the distance function, but the same procedures to compute the bounds would still hold.

The bounding relation (22) has only been proved for linear elastic behavior in small strains. It could easily be extended to nonlinear elasticity or viscoplasticity, within the same small strain framework, as the classical minimal potential or complementary energy principles they rely on also apply to such behaviors derived from single convex stress or strain potentials. Extensions to more general behaviors or kinematics such as finite elasticity or elastoplasticity are left for future work.

At last, since the VOR bounds converge quickly towards the effective behavior, they can be used to evaluate the RVE size associated with a specific effective property for a given random composite. In fact, there are many ways to define a RVE size (e.g.
Gitman et al., 2007; Kanit et al., 2003; Pelissou et al., 2009; Willot and Jeulin, 2009). One possible way - often adopted in the context of mean fields homogenization theories - is to consider that the RVE size is reached when the apparent behavior of any VE and associated with any macrohomogeneous BC is sufficiently close to the effective behavior. According to this viewpoint, the determination of the RVE size requires to investigate not only the mean values of the apparent behaviors, i.e. the VOR bounds, but also their statistical fluctuations. This requires to perform a statistical study of the apparent behavior as presented in by Salmi et al. (2012).

\section{References}

Beran, M., 1968. Statistical Continuum Theories. John Wiley, New York.

Bilger, N., Auslender, F., Bornert, M., Michel, J.-C., Moulinec, H., Suquet, P., Zaoui, A., 2005. Effect of a non uniform distribution of voids on the plastic response of voided materials: a computational and statistical analysis. Int. J. Solids Struct. 42, 517-538.

Bornert, M., 2001. Homogénéisation des milieux aléatoires: bornes et estimations In: Bornert, M., Bretheau, T., Gilormini, P. (Eds.), Homogénéisation en mécanique des matériaux 1: Matériaux aléatoires élastiques et milieux périodiques. Hèrmes Science Publication, pp, pp. 133-221.

Bornert, M., Stolz, C., Zaoui, A., 1996. Morphologically representative pattern-based bounding in elasticity. J. Mech. Phys. Solids 44, 307-331.

Casella, G., Berger, R., 2001. Statistical Inference. Duxbury, United States.

CEA, 2003. Cast3m. http://www-cast3m.cea.fr/.

Christensen, R.M., Lo, K.H., 1979. Solution for effective shear properties in three phase sphere and cylinder models. J. Mech. Phys. Solids 27, 315-330.

Danielsson, M., Parks, D., Boyce, M., 2007. Micromechanics, macromechanics and constitutive modeling of the elasto-viscoplastic deformation of rubbertoughened glassy polymers. J. Mech. Phys. Solids 55, 533-561.

Doghri, I., Adam, L., Bilger, N., 2010. Mean-field homogenization of elastoviscoplastic composites based on a general incrementally affine linearization method. Int. J. Plast. 26, 219-238.

Eshelby, J.D., 1957. The determination of the elastic field of an ellipsoidal inclusion, and related problems. Proc. R. Soc. Lond. A A421, 376-396.

Gitman, I., Askes, H., Sluys, L., 2007. Representative volume: existence and size determination. Eng. Fracture Mech. 74, 2518-2534.

Gusev, A., 1997. Representative volume element size for elastic composites: a numerical study. J. Mech. Phys. Solids 45 (9), 1449-1459.

Hashin, Z., 1962. The elastic moduli of heterogeneous materials. J. Appl. Mech. 29, 143-150.

Hashin, Z., Rosen, B.W., 1964. The elastic moduli of fiber-reinforced materials. J. Appl. Mech. 31, 223-232.

Hashin, Z., Shtrikman, S., 1963. A variational approach to the theorie of the elastic behavior of multiphase material. J. Mech. Phys. Solids 11, 127-140.

Huet, C., 1990. Application of variational concepts to size effects in elastic heterogeneous bodies. J. Mech. Phys. Solids 38, 813-841.

Jiang, M., Alzebdeh, K., Jasiuk, I., Ostoja-Starzewski, M., 2001. Scale and boundary conditions effects in elastic properties of random composites. Acta Mech. 148 (1-4), 63-78.

Kanit, T., Forest, S., Galliet, I., Mounoury, V., Jeulin, D., 2003. Determination of the size of the representative volume element for random composites: statistical and numerical approach. Int. J. Solids Struct. 40, 3647-3679.

Kroner, E., 1958. Berechnung der elastischen konstanten des vielkrilstalls aus den konstanten des einkristalls. Z. Phys. 151, 504-518.

Lahellec, N., Suquet, P., 2004. Nonlinear composites: a linearization procedure, exact to second-order in contrast and for which the strain-energy and affine formulations coincide. C.R. Mec. 332, 693-700.

Lahellec, N., Suquet, P., 2007. On the effective behavior of nonlinear inelastic composites: I Incremental variational principles. J. Mech. Phys. Solids 55, 19321963.

Masson, R., Zaoui, A., 1999. Self-consistent estimates for the rate-dependent elastoplastic behaviour of polycrystalline materials. J. Mech. Phys. Solids 47 1543-1568.

Mori, T., Tanaka, K., 1973. Average stress in matrix and average elastic energy of materials with misfitting inclusions. Acta Metall. 21, 597-629.

Nemat-Nasser, S., Hori, M., 1993. Micromechanics: overall Properties of Heterogenous Materials. North Holland, Amsterdam.

Ostoja-Starzewski, M., 1998. Random field models of heterogeneous materials. Int J. Solids Struct. 35, 2429-2455.

Ostoja-Starzewski, M., 2006. Material spatial randomness: from statistical to representative volume element. Prob. Eng. Mech. 21, 112-132.

Papanicolaou, G., Varadhan, S., 1978. Boundary Value Problems with Rapidly Oscillating Random Coefficients. North-Holland, Amsterdam, pp. 835-873.

Pelissou, C., Baccou, J., Monerie, Y., Perales, F., 2009. Determination of the size of the representative volume element for random quasi-brittle composites. Int. J. Sol. Struct. 46 (14-15), 2842-2855.

Ponte Castañeda, P., 2002. Second-order homogeneisation estimates for nonlinear composites incorporating field fluctuations: I- theory. J. Mech. Phys. Solids 50, 737-757. 
Ponte Castañeda, P., Suquet, P., 1997. Nonlinear composites. Advances in Applied Mechanics 34, 171-212, 212a, 212b, 212c, 212d, 213-302.

Ponte Castañeda, P., Willis, J., 1995. The effect of spatial distribution on the effective behavior of composite materials and cracked media. J. Mech. Phys. Solids 43 (12), 1919-1951.

Ranganathan, S., Ostoja-Starzewski, M., 2008. Scaling function, anisotropy and the size of RVE in elastic random polycrystals. J. Mech. Phys. Solids 56, 2773-2791.

Reuss, A., 1929. Calculation of the flow limits of mixed crystals on the basis of the plasticity of mono-crystals. Z. Angew. Math. Mech 9, 49-58.

Rintoul, M., Torquato, S., 1997. Reconstruction of the structure of dispersions. J. Colloid Interface Sci. 186 (2), 467-476.

Sab, K., 1992. On the homogenization and the simulation of random materials. Eur. J. Mech. A/Solids 11, 585-607.

Salmi, M., 2011. Homogénéisation des composites linéaires: étude de la variabilité des comportements. Ph.D. thesis, Université Blaise Pascal. Clermont-Ferrand (France).

Salmi, M., Auslender, F., Bornert, M., Fogli, M., 2012. Various estimates of representative volume element sizes based on a statistical analysis of the apparent behavior of random linear composites. Comptes Rendus Mécanique, in press.
Sanchez-Palencia, E., Zaoui, A. (Eds.), 1987. vol. 272. Springer-Verlag, New York. Schoberl, J., Gerstmayr, H., Gaisbauer, R., 2003. Netgen - Automatic mesh generator. http://www.hpfem.jku.at/netgen/.

Stolz, C., Zaoui, A., 1991. Analyse morphologique et approches variationnelles du comportement d'un milieu élastique hétérogène. C.R. Acad. Sci. Paris, II 312, $143-150$.

Voigt, W., 1889. über die beziehung zwischen den beiden elasticitätsconstanten isotroper körper. Ann. Phys. (Leipzig) 38, 573-587.

Walpole, L., 1981. Elastic Behavior of Composite Materials: Theoretical Foundations. vol. 21. Academic Press, New York, pp. 160-242.

Willis, J., 1977. Bounds and self-consistent estimates for the overall properties of anisotropic composites. J. Mech. Phys. Solids 25, 185-202.

Willis, J., 1981. Variational and related methods for the overall properties of composites. Adv. Appl. Mech. 21, 1-78.

Willot, F., Jeulin, 2009. Elastic behavior of composites containing boolean random sets of inhomogeneities. Int. J. Eng. Sci. 47, 313-324.

Willot, F., Pellegrini, Y.-P., Ponte Castañeda, P., 2008. Localization of elastic deformation in strongly anisotropic, porous, linear materials with periodic microstructures: exact solutions and dilute expansions. J. Mech. Phys. Solids 56 (4), 1245-1268. 